\title{
Decay of Solutions of Hyperbolic Systems of Conservation Laws with a Convex Extension
}

\author{
Ronald J. DiPerna \\ Communicated by C. DAFERMOS
}

\section{Introduction}

We study solutions of the Cauchy problem for strictly hyperbolic systems of conservation laws of the form

$$
\frac{\partial}{\partial t} U+\frac{\partial}{\partial x} F(U)=0, \quad-\infty<x<\infty, \quad t>0 .
$$

Here $U=U(x, t)$ takes on values in $R^{n}$ and $F$ is a smooth nonlinear mapping from $R^{n}$ to $R^{n}$. The condition of strict hyperbolicity requires the Jacobian $F^{\prime}$ of $F$ to have $n$ real and distinct eigenvalues

$$
\lambda_{1}(U)<\cdots<\lambda_{n}(U)
$$

We shall suppose in addition that each characteristic field of the system (1.1) is either genuinely nonlinear in the sense of LAX [17], that is

$$
r_{j} \cdot \nabla \lambda_{j} \neq 0
$$

or linearly degenerate in the sense of LAX [17], that is

$$
r_{j} \cdot \nabla \lambda_{j} \equiv 0 \text {. }
$$

Here $r_{j}=r_{j}(U)$ denotes the right eigenvector of $F^{\prime}$ which corresponds to $\lambda_{j}$.

The existence of solutions to systems with the above structure has been established by GLimm [9]. The existence theory of Glimm treats the Cauchy problem with initial data having small total variation and constructs solutions as the limit of a sequence of difference approximations. The space of functions of bounded variation is a natural invariant space for the solution operator of a system of conservation laws. While the theory is developed in the context of genuinely non-linear systems [9], only very minor modifications are necessary for a generalization to systems which admit linearly degenerate characteristic fields. We refer the reader to [16] for an extension of Glimm's method to an even wider class of systems.

In this paper we study the decay problem for the class $K$ of systems which possess a generalized entropy in the sense of LAX [18]. As observed by FRIEDRICHS \& LAX [8], most of the conservative systems of mathematical physics are 
endowed with a generalized entropy. For convenience in describing our results we partition the class $K$ into two subclasses. The first subclass $K_{1}$ consists of systems which admit both genuinely nonlinear and linearly degenerate characteristic fields. The subclass $K_{1}$ contains the equations of gas dynamics $(n=3)$ in both relativistic and nonrelativistic form and the Lundquist equations of magneto-fluid-dynamics $(n=7)$. The second subclass $K_{2}$ consists of genuinely nonlinear systems, i.e. systems all of whose characteristic fields are genuinely nonlinear. The subclass $K_{2}$ contains the isentropic equations of gas dynamics $(n=2)$ in both relativistic and nonrelativistic form, the equations of shallow water waves $(n=2)$ and the equations of finite amplitude plane elastic waves superimposed on a constant state of strain $(n=6)$. It has been shown by JoHN [15] that for classical materials the latter system is genuinely nonlinear provided that the wave front does not contain a principle direction of strain. The subclass $K_{2}$ also contains the class of systems of two equations introduced by SMOLLER \& JOHNSON [24] and the class of genuinely nonlinear symmetric hyperbolic systems ( $n$ arbitrary). The aforementioned systems are recorded for reference in Section 2 along with their associated generalized entropies.

Throughout this paper we assume for simplicity that the equation of state which enters the above fluid systems is that of a polytropic gas with adiabatic exponent $\gamma$ satisfying $1<\gamma<5 / 3$. This assumption guarantees the existence of solutions to the Riemann problem and the construction of the Glimm difference approximations. Section 2 also contains a brief sketch of LAX's method of constructing solutions to the Riemann problem [17] together with an outline of Glimm's difference scheme and associated functionals.

The theory of decay for genuinely nonlinear systems of conservation laws has been developed by GlimM \& LAX [10]. The Glimm-Lax theory is developed in the context of systems of two conservation laws and treats the Cauchy problem with initial data having small oscillation. The following results were established in [10]. In the case of initial data with compact support all solutions decay to zero in the total variation norm, uniformly at the rate $t^{-1 / 2}$ :

$$
T V_{x} U(x, t) \leqq \text { const. } t^{-1 / 2} \text {. }
$$

In the case of periodic initial data the total variation per period decays uniformly at the rate $t^{-1}$. Furthermore, the Glimm-Lax theory demonstrates that the primary mechanisms of decay in genuinely nonlinear systems are the spreading of rarefaction waves and the interaction of shock waves and rarefaction waves of the same characteristic field. Since these mechanisms also prevail in a genuinely nonlinear system of $n$ conservation laws we expect that the Glimm-Lax theory will generalize to such systems and will yield a uniform algebraic decay rate for solutions whose initial data have sufficiently small total variation. With regard to the theory of special solutions we refer the reader to GREENBERG [12] for a decay analysis of a class of periodic solutions to the particular system $(n=2)$ generated by the quasilinear wave equation and to GREENBERG [13] for an analysis of a class of initial-boundary value problems for the same system.

In terms of the large-time behavior of the solution, the principle feature which distinguishes a genuinely nonlinear system from a system which admits linearly degenerate characteristic fields is the existence of generalized contact disconti- 
nuities. Generalized contact discontinuties constitute the elementary waves of the linearly degenerate fields while shock waves, rarefaction waves and compression waves constitute the elementary waves of the genuinely nonlinear fields. However, in contrast to genuinely nonlinear fields, neither the process of spreading of waves nor the process of interaction and cancellation of waves occurs in linearly degenerate fields. Consequently, only certain distinguished components of the solution decay to zero in a system of conservation laws which admits linearly degenerate characteristic fields. Simple examples can be constructed in which generalized contact discontinuities do not decay to zero with large time. We shall describe such examples below.

The main purpose of this paper is to study the decay of solutions to systems of conservation laws which admit linearly degenerate fields. We treat the Cauchy problem with initial data having compact support and carry out our analysis for solutions constructed by the Glimm difference scheme. The initial data which we consider need not have small oscillation. We derive three main estimates which govern the decay of solutions, namely Lemmas 3.1, 4.2, and 5.2. The first estimate is based on the concept of generalized entropy introduced by LAX [18] and on a method of constructing "normalized" entropies given by DAFERMOs [3]. The first estimate establishes a relationship between the total entropy of the solution and the magnitudes of the individual shock waves in the solution. The second and third estimates are based on the geometry of the elementary waves of the solution and, respectively, establish relationships between the magnitudes of individual shock waves and the oscillation of the solution and between the oscillation of the solution and its total variation. The main tool used in the derivation of the second and third estimates is the theory of generalized characteristics developed by GLImm \& LAX [10].

For simplicity we shall first describe our results in the case of initial data having small total variation. Let $U=U(x, t)$ be a solution of a system in class $K_{1}$ whose initial data are constant outside a bounded interval. Let the indices $\alpha_{1}, \ldots, \alpha_{m}$ reference the linearly degenerate fields, i.e.

$$
r_{k} \cdot \nabla \lambda_{k} \equiv 0, \quad k=\alpha_{1}, \ldots, \alpha_{m} .
$$

Suppose $\phi=\phi(U)$ is a Riemann invariant for all of the linearly degenerate fields, so that

$$
r_{k} \cdot \nabla \phi \equiv 0, \quad k=\alpha_{1}, \ldots, \alpha_{m} .
$$

If the total variation of the initial data is sufficiently small, then the solution $U$ satisfies the decay law

$$
\lim _{t \rightarrow \infty} T V_{x} \phi\{U(x, t)\}=0 .
$$

We recall that a Riemann invariant for a given linearly degenerate field is necessarily continuous across the generalized contact discontinuities which exist in that field. Consequently the function $\phi$ recognizes only the elementary waves of the genuinely nonlinear fields, namely shock waves, rarefaction waves and compression waves, and the decay law (1.2) can be interpreted as the statement that the total strength of all elementary waves other than contact discontinuities 
decays to zero with large time. It would be interesting to determine whether or not $\phi$ actually decays at an algebraic rate.

The decay law (1.2) applies to systems of fluid flow in class $K_{1}$ in the following way. For the nonisentropic equations of fluid dynamics in both nonrelativistic and relativistic form, the first and third characteristic fields are genuinely nonlinear while the second is linearly degenerate. The velocity and pressure of the fluid are precisely the fluid variables which qualify as Riemann invariants for the second field. As such they both decay to a constant state in the total variation norm. An example of a solution in which the remaining fluid variables do not decay can be constructed as follows. Consider a solution which consists of precisely three constant states separated by two contact discontinuities propagating parallel to one another. In such a solution the velocity and pressure are identically constant, while the density, internal energy, etc. maintain constant values in the strip between the contact discontinuities which are in general distinct from their values outside the strip.

In the case of magneto-fluid-dynamics the second, fourth and sixth characteristic fields are linearly degenerate. We recall that discontinuities in the second and fourth fields are Alfven waves and discontinuities in the fourth field are (classical) contacts. The $x$-components of the fluid (vector) velocity and the pressure are precisely the quantities which qualify as Riemann invariants for all of the linearly degenerate fields. As such they both decay to a constant state in the total variation norm. In a similar way solutions can be constructed in which all of the remaining fluid and magnetic variables fail to decay due to the presence of generalized constant discontinuities.

The analysis used to establish the decay law (1.2) applies equally well to genuinely nonlinear systems of $n$ conservation laws and yields the following theorem in that case. Let $U$ be a solution to a system in class $K_{2}$ whose initial data are constant outside a bounded interval. If the initial data have sufficiently small total variation then the solution $U$ satisfies the decay law

$$
\lim _{t \rightarrow \infty} T V_{x} U(x, t)=0
$$

In this paper we also study the decay of solutions with large initial data. We recall that solutions of the Cauchy problem with initial data having only finite total variation can be constructed in a natural way by establishing a uniform bound on the total variation of the Glimm difference approximations $U_{h}$, for instance

$$
T V_{x} U_{h}(x, t) \leqq \text { const., }
$$

where the constant depends only on the total variation of the initial data. It follows by a compactness argument based on Helly's theorem [9] that the approximate solutions converge to an exact solution which assumes the given initial data. In the case of initial data having small total variation the uniform bound (1.4) was established by GLIMM with the aid of certain nonlinear functionals defined on the approximate solutions $U_{h}$. Using various modifications of the GLIMM functionals, the uniform bound (1.4) has been established for certain systems of conservation laws without the restriction of small data [1], [5], [20], 
[21], [22]. In particular NisHida [15] proved the uniform bound (1.4) for the isothermal (model) equations of gas dynamics,

$$
\begin{gathered}
\frac{\partial}{\partial t} u+\frac{\partial}{\partial x} p(v)=0, \quad p(v)=\text { const. } v^{-1} \\
\frac{\partial}{\partial t} v-\frac{\partial}{\partial x} u=0
\end{gathered}
$$

with arbitrary initial data having finite total variation and in so doing gave the first example of a system of conservation laws for which the Cauchy problem can be solved with large data. For the isentropic equations of a polytropic gas, $p(v)=$ const. $v^{-\gamma}, \gamma>1$, NiSHIDA \& SMOLLER [22] obtained the uniform bound (1.4) under the following condition on the initial data:

$$
(\gamma-1) T V U(x, 0) \leqq \text { const. }
$$

where the constant is independent of the adiabatic exponent $\gamma$. Recently, Liu [20] has constructed solutions for the equations of a polytropic gas $(n=3)$ under the Nishida-Smoller condition with $1<\gamma \leqq 5 / 3$. As a corollary of a general theorem which we shall state below it can be shown that the aforementioned systems satisfy the above decay laws under the associated conditions on their initial data. Furthermore, it follows as a corollary of the Glimm-Lax theory that the solutions of the isothermal and isentropic equations of gas dynamics also decay at the algebraic rate $t^{-1 / 2}$ in the case of large initial data. We expect (but do not show) that the decay rate for the latter equations is uniform, i.e. depends only on the total variation of the initial data.

In addition to the systems above there exist two special classes of systems for which globally defined solutions have been constructed with arbitrary initial data having finite total variation [1], [5]. These special classes are motivated by but (apparently) do not contain physical equations. It would be interesting to determine whether or not their member systems possess a generalized entropy.

The most general set of hypotheses under which we establish the decay laws (1.2) and (1.3) is the following (cf. Theorems 5.1 and 5.2). First, the initial data is constant outside a bounded interval and has finite total variation. Second, the total amount of wave interaction in the Glimm difference approximations $U_{h}$ is uniformly bounded in $h$. We note that this condition is only slightly stronger than the condition (1.4) that the total variation of difference approximations $U_{h}$ be uniformly bounded (cf. Section 2). In the case of systems for which the smallness condition on the variation has been relaxed the uniform bound can be established in a straightforward way. Third, the eigenvalues $\lambda_{j}$ are strictly separated on the range $\Omega$ of the sequence of Glimm difference approximations:

$$
\max \left\{\lambda_{j}(U): U \in \Omega\right\}<\min \left\{\lambda_{j+1}(U): U \in \Omega\right\},
$$

where

$$
\Omega \supset\left\{U_{h}(x, t): h>0,-\infty<x<\infty, t>0\right\} .
$$

We note that condition (1.5) automatically holds in the case of initial data with small total variation since the system is strictly hyperbolic. We also recall that 
there exist a number of systems for which the eigenvalues $\lambda_{j}$ are strictly separated on an arbitrary compact set and consequently for which the third hypothesis is an immediate consequence of the second. In particular, condition (1.5) is satisfied by the Lagrangian form of the following systems: the isothermal and isentropic equations of gas dynamics $(n=2)$, the equations of shallow water waves $(n=2)$ and the general equations of gas dynamics $(n=3)$. However, condition (1.5) represents a restriction on the oscillation of the solution for the equations of magneto-fluid-dynamics and the equations of finite amplitude plane elastic waves.

Our analysis of decay is based on three main estimates each of which expresses one principle of decay governing the solution. The first estimate expresses the fact that a solution with uniformly bounded total entropy can not contain large shock waves at arbitrarily large times. More precisely, we show that the maximum strength of all shock waves which exist in the solution at a given time $t$ decays to zero as $t$ approaches infinity. This phenomenon is essentially a consequence of the boundedness of total entropy and the boundedness of the total amount of wave interaction (cf. Section 3).

The second estimate expresses the fact that a solution cannot support at arbitrarily large times both large oscillations and weak shock waves. We show that the oscillation of the solution at a fixed time $t=\tau$ is bounded by the sum of the following quantities: the maximum strength of all shock waves which exist for all times $t \geqq \tau$ in the future, the total amount of wave interaction and cancellation which occurs at all times in the future, and a quantity which measures the degree of coupling of the characteristic fields. In the case of a system of conservation laws which admits linearly degenerate characteristic fields, the oscillation of the solution is replaced in the second estimate by the oscillation of the distinguished components of the solution. The basis of the second principle is the following. Large oscillations of the solution in a given genuinely nonlinear field exist either as a consequence of one or more large shock waves or compression waves or as a consequence of a large number of weak shock waves. In the latter case the joint action of the waves is similar to a single compression wave which eventually breaks and produces a single large shock wave. The only mechanisms which may disturb this breaking process are those of wave interaction and cancellation. It is essentially for this reason that interaction and cancellation terms enter the second estimate. Using the first and second estimates it can be shown that the oscillation of solutions of systems in class $K_{2}$ and the oscillation of the distinguished components of solutions of systems in class $K_{1}$ decay to zero with large time (cf. Section 4).

The third estimate expresses the fact that a solution cannot support both large total variation and small oscillation at arbitrarily large times. The third estimates provides a bound on the total variation of the solution or its distinguished components at time $t=\tau$ in terms of the following quantitites: the respective oscillations at time $t=\tau$, the total amount of wave interaction and cancellation which occurs at all times $t \geqq \tau$ in the future, and a quantity which measures the degree of coupling of the characteristic fields. The basis of the third principle is roughly the following. In a genuinely nonlinear field the only way a solution with small oscillation can achieve a large total variation is by 
admitting a large number of weak shock waves and/or compression waves alternating with a nearly equal number of rarefaction waves. In a solution with this structure the waves must either interact or cancel. We establish the decay laws (1.2) and (1.3) using the third estimate and the decay of the oscillation (cf. Section 5).

\section{Preliminaries}

\section{§1. The Glimm Difference Approximations}

Consider a system of conservation laws (1.1) each of whose characteristic fields is either genuinely nonlinear or linearly degenerate in the sense of LAX [7]. The Glimm difference scheme constructs solutions to the Cauchy problem as the limit of a sequence of approximate solutions each of which consists locally of solutions of the Riemann problem. We recall that the Riemann problem is the Cauchy problem with initial data of the form

$$
U(x, 0)=\left\{\begin{array}{ll}
U_{l} & x<0 \\
U_{r} & x>0
\end{array},\right.
$$

where $U_{l}$ and $U_{r}$ are constant states. The Riemann problem for systems with the above structure has been solved by LAX [17] in the case where the initial data $U_{l}$ and $U_{r}$ are sufficiently close. The Riemann problem with arbitrary initial data has been solved only for certain special systems of equations. We refer the reader to SMOLLER [23] for a study of a broad class of systems of two conservation laws which includes the isentropic equations of gas dynamics and the equations of shallow water waves, to LIU [20] for a study of the general equations of gas dynamics and to Gogosov [11] for a study of the equations of magneto-fluiddynamics. To the knowledge of this author, the Riemann problem with arbitrary data is presently open for the relativistic equations of gas dynamics and the equations of finite amplitude plane elastic waves. The aforementioned fluid dynamical systems are recorded for reference in Subsection 2.

Before describing the Glimm difference approximations we shall recall the structure of the solution of the Riemann problem. The Riemann problem admits a self-similiar solution $U=U(x / t)$ which consists in general of $n$ elementary waves, one corresponding to each characteristic field and any two separated by a constant state. The solution takes on constant values $U_{0}=U_{l}, \ldots, U_{j}, \ldots, U_{N}=U_{r}$ in consecutive sectors of the form

$$
\Omega_{j}=\left\{(x, t): a_{j} t<x<b_{j} t\right\} .
$$

If the $j^{\text {th }}$ characteristic field is genuinely nonlinear the sectors $\Omega_{j-1}$ and $\Omega_{j}$ are separated by either a $j$-shock or a centered $j$-rarefaction wave, cf. [17]. If the $j^{\text {th }}$ field is linearly degenerate then $\Omega_{j-1}$ and $\Omega_{j}$ are separated by a $j$-contact discontinuity, i.e., a discontinuity whose speed of propagation coincides with the characteristic speed $\lambda_{j}$ on both of its sides. Throughout this paper we shall assume that the initial data of all Riemann problems under consideration are such that there exists a solution $U=U(x / t)$ with the above structure.

The solution of the Riemann problem is constructed with the aid of a family of wave curves [17]. A collection of $n$ curves is associated with each constant 
state $U_{0}$. The $j^{\text {th }}$ wave curve,

$$
\begin{gathered}
U_{j}=U_{j}\left(\varepsilon ; U_{0}\right) \\
U_{j}\left(0, U_{0}\right)=U_{0},
\end{gathered}
$$

forms a one-parameter family of states $U_{j}(\varepsilon)$ which can be connected to $U_{0}$ on the right by an elementary $j$-wave. If the $j^{\text {th }}$ field is genuinely nonlinear the curve $U_{j}(\varepsilon)$ consists of two smooth branches: positive values of the wave parameter $\varepsilon$ yield states $U_{j}(\varepsilon)$ which can be connected to $U_{0}$ through a $j$-rarefaction wave while negative values yield states $U_{j}(\varepsilon)$ which can be connected to $U_{0}$ through a $j$-shock wave. The union of the two branches forms a $C^{2}$ curve passing through $U_{0}$. Indeed, the local geometry of the $j^{\text {th }}$ wave curve of a genuinely nonlinear field is given by

$$
\begin{aligned}
& \dot{U}_{j}=r_{j}\left(U_{j}\right) \quad \text { if } \varepsilon \geqq 0 \\
& \dot{U}_{j}=r_{j}\left(U_{j}\right)+o\left(\varepsilon^{2}\right) \quad \text { if } \varepsilon<0 \\
& \ddot{U}_{j}=\left(r_{j} \cdot \nabla\right) r_{j}\left(U_{j}\right) \quad \text { if } \varepsilon \geqq 0 \\
& \ddot{U}_{j}=\left(r_{j} \cdot \nabla\right) r_{j}\left(U_{j}\right)+o(\varepsilon) \quad \text { if } \varepsilon<0 .
\end{aligned}
$$

In the equation (2.1) we have adopted the standard normalization of the right eigenvectors $r_{j}$ corresponding to the genuinely nonlinear fields, namely

$$
r_{j} \cdot \nabla \lambda_{j} \equiv 1
$$

If the $j^{\text {th }}$ characteristic field is linearly degenerate the $j^{\text {th }}$ wave curve $U_{j}=U_{j}\left(\varepsilon ; U_{0}\right)$ is a smooth curve,

$$
\begin{gathered}
\dot{U}_{j}=r_{j}\left(U_{j}\right) \\
U_{j}\left(0, U_{0}\right)=U_{0},
\end{gathered}
$$

passing through $U_{0}$ that consists entirely of states which can be connected to $U_{0}$ through a $j$-contact discontinuity.

The solution of the Riemann problem is constructed by forming an $n$-fold composition of the $j$-wave curves, $j=1,2, \ldots, n$. This composition yields an $n$-parameter family of states which can be connected to a fixed state (say) $U_{l}$ through a sequence of $n$ elementary waves. The implicit function theorem guarantees that a small neighborhood of zero in parameter space is mapped in a one-to-one fashion onto a neighborhood of $U_{l}$; the Jacobian of the mapping is non-zero at the origin since the derivative with respect to the $k^{\text {th }}$ parameter is the eigenvector $r_{k}$, cf. [17]. In the case of arbitrary Riemann data a global analysis of the geometry of the wave curves is required to show that the mapping is onto.

Lastly we recall that the continuation of a $j$-rarefaction wave curve to negative values of the parameter $\varepsilon$ constitutes a one-parameter family of states which can be connected to $U_{0}$ through a $j$-compression wave. Accordingly we shall refer to the curve $U_{j}(\varepsilon)$ defined by

$$
\begin{aligned}
\dot{U}_{j}(\varepsilon) & =r_{j}\left(U_{j}(\varepsilon)\right), \quad \varepsilon \leqq 0 \\
U_{j}(0) & =U_{0},
\end{aligned}
$$

as the $j$-compression wave curve through $U_{0}$. 


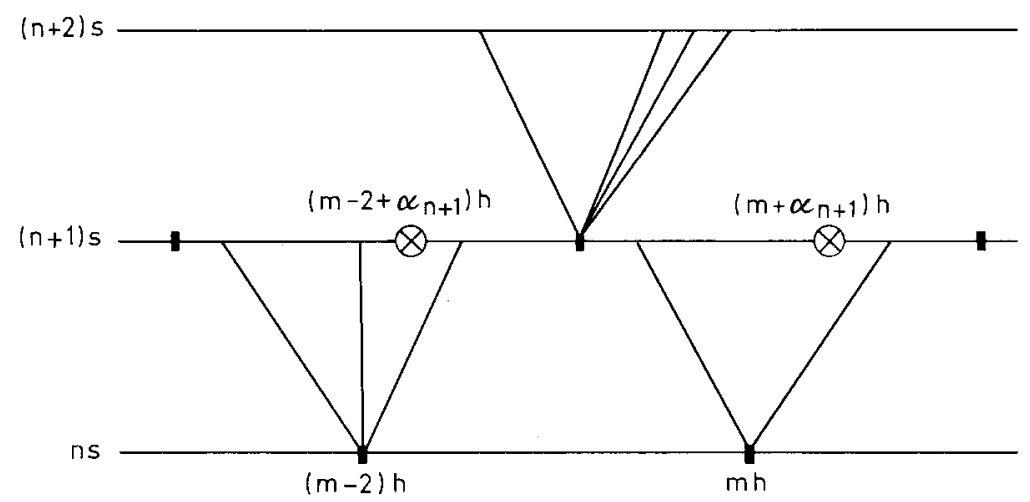

Fig. 1

We shall now recall the structure of the Glimm difference approximations. An approximate solution $U_{h}$ is an exact solution in strips $S_{n}$ of the form

$$
S_{n}=\{(x, t): n s \leqq t<(n+1) s\}
$$

where $s$ denotes the mesh-length in time. At time $t=n s, U_{h}$ is a piecewise constant function of $x$ whose discontinuities are contained in the set $\{m h: m+n$ even $\}$. The discontinuities of $U_{h}(\cdot, n s)$ generate Riemann problems whose solutions constitute the continuation of $U_{h}$ into $S_{n}$. At time $t=(n+1) s$ the approximate solution $U_{h}$ is redefined as a piecewise constant function through the use of a random equidistributed parameter $\alpha_{n} \varepsilon(-1,1)$; in the interval $(m-1) h<x<(m+1) h$, the approximate solution $U_{h}$ is defined to be the constant state

$$
U_{h}\left\{\left(m+\alpha_{n+1}\right) h,(n+1) s-0\right\}
$$

(see figure 1). At time $t=(n+1) s$ the Riemann problems generated at $x=m h$, $m+(n+1)$ even, are solved with the initial data

$$
\begin{aligned}
& U_{l}=U_{h}\left\{\left(m-2+\alpha_{n-1}\right) h,(n+1) s-0\right\} \\
& U_{r}=U_{h}\left\{\left(m+\alpha_{n+1}\right) h,(n+1) s-0\right\} .
\end{aligned}
$$

The solutions of these Riemann problems extend the approximate solution $U_{h}$ into the strip $S_{n+1}$.

In order to establish the relative compactness of the family of approximate silutions it is sufficient to prove a uniform estimate on the total variation of $U_{h}$ of the form

$$
T V_{x} U_{h}(x, t) \leqq \text { const., }
$$

where the constant is independent of $h$ and $t$. In the case of initial data with small total variation, the uniform bound (2.3) was establish by Glimm [9] with the aid of certain approximate conservation laws which govern the magnitudes of interacting elementary waves. We shall recall these relations presently. The random mesh points

$$
a_{m, n}=\left\{\left(m+\alpha_{n}\right) h, n s\right\}
$$




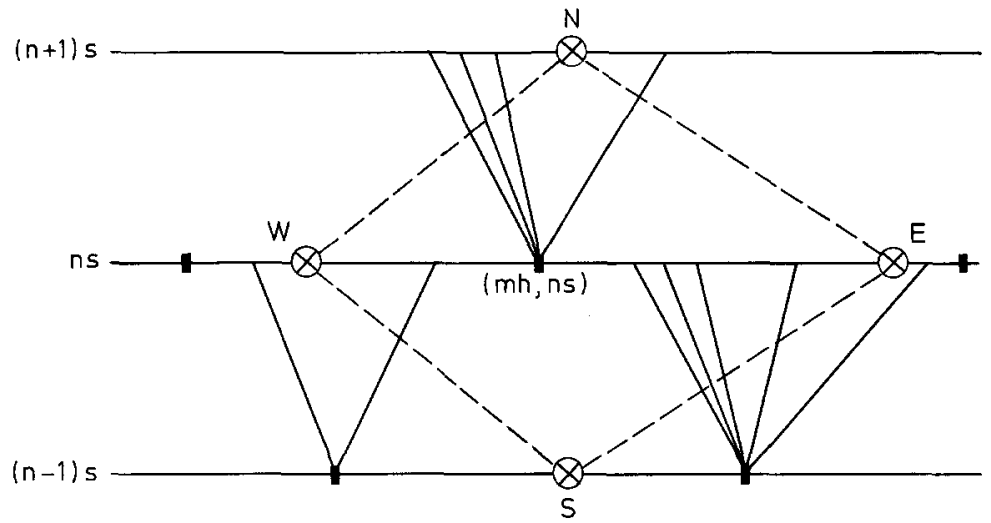

Fig. 2

partition the half-plane $t>0$ into a countable number of diamond-shaped domains $\Delta_{n, m}$ which are centered at the points $(m h, n s)$ and which are defined by their vertices

$$
\begin{aligned}
S & =\left\{\left(m+\alpha_{n-1}\right) h,(n-1) s\right\}, & N & =\left\{\left(m+\alpha_{n+1}\right) h,(n+1) s\right\} \\
W & =\left\{\left(m-1+\alpha_{n}\right) h, n s\right\}, & & E=\left\{\left(m+1+\alpha_{n}\right) h, n s\right\}
\end{aligned}
$$

(see figure 2). The waves which are incoming with respect to the diamond $\Delta_{n, m}$ are those which originate at time $t=(n-1) s$ at $x$-coordinates $(m-1) h$ and $(m+1) h$. The outgoing waves originate at time $t=n s$ at $x$-coordinate $m h$. There are at most $2 n$ incoming waves and at most $n$ outgoing waves.

The conservation laws for waves relate the magnitude of the outgoing waves of an interaction to the magnitudes of the incoming waves. One of the standard measurements of wave magnitude is the wave parameter $\varepsilon$ : the elementary $j$-wave which connects the state $U_{0}$ to the state $U_{j}\left(\varepsilon ; U_{0}\right)$ is said to have magnitude $\varepsilon$. For weak waves an equivalent measurement can be obtained by first selecting a function $w$ which satisfies

$$
r_{j} \cdot \nabla w=1
$$

and then defining the magnitude of an elementary $j$-wave as

$$
w\left\{U_{j}(\varepsilon)\right\}-w\left\{U_{0}\right\}
$$

Using (2.4) we can easily verify that

$$
w\left\{U_{j}(\varepsilon)\right\}-w\left\{U_{0}\right\}=\varepsilon+O\left(\varepsilon^{3}\right) .
$$

We note that in general one can only guarantee the existence of solutions to equation (2.4) in the neighborhood of a given state $U_{0}$. However, we observe that the eigenvalues $\lambda_{j}$ provide globally defined solutions for those indices $j$ which correspond to genuinely nonlinear characteristic fields. Furthermore, the Lax shock conditions imply that this measurement of $j$-waves is equivalent to the Euclidean distance in the sense that

$$
\text { const. }\left\|U_{j}(\varepsilon)-U_{0}\right\| \leqq\left|\lambda_{j}\left\{U_{j}(\varepsilon)\right\}-\lambda_{j}\left(U_{0}\right)\right| \leqq \text { const. }\left\|U_{j}(\varepsilon)-U_{0}\right\| .
$$




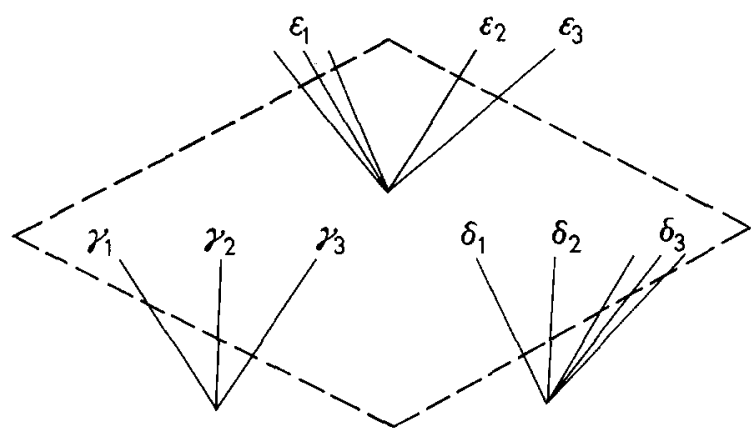

Fig. 3

The measurement of the magnitude of the elementary waves of a genuinely nonlinear field through the corresponding eigenvalues proves to be useful in our analysis of the decay of solutions.

We shall now state the conservation laws for waves. Let $\varepsilon_{j}=\varepsilon_{j}(\Delta)$ denote the magnitude of the outgoing $j$-wave in the diamond $\Delta$ and let $\gamma_{j}=\gamma_{j}(\Delta)$ and $\delta_{j}=\delta_{j}(\Delta)$ denote the magnitudes of the incoming $j$-waves (see Figure 3). Let $Q(\Delta)$ denote the total amount of wave interaction in the diamond $\Delta$, that is

$$
Q(\Delta)=\Sigma\left\{\left|\gamma_{i}\right|\left|\delta_{j}\right|: \gamma_{i} \text { and } \delta_{j} \text { approach }\right\}
$$

Here the waves $\gamma_{i}$ and $\delta_{j}$ are said to approach if the following holds: either $i>j$ and $\gamma_{i}$ lies to the left of $\delta_{j}$ or $i=j$ and not both $\gamma_{i}$ and $\delta_{j}$ are rarefaction waves or contact discontinuities.

The magnitudes of the elementary waves of a given characteristic field are conserved up to linear terms while the deviation from linearity is governed by an error term whose order is bounded by the total amount $Q(\Delta)$ of wave interaction in $\Delta$ :

$$
\varepsilon_{j}=\gamma_{j}=\delta_{j}+O\{Q(\Delta)\}
$$

The approximate conservation laws (2.6) were established by GLimm [9] for genuinely nonlinear systems. However, the proof also applies to systems with degenerate fields; the local geometry of the wave curves of a linearly degenerate field is identical with that of a genuinely nonlinear field up to third order terms in the wave parameter $\varepsilon$ (cf. (2.2) and (2.3)). The interaction term $Q(\Delta)$ does not contain either the product of magnitudes of contact discontinuities of the same field or the product of magnitudes of rarefaction waves of the same field be cause the magnitudes of such pairs of waves are additive under binary interactions.

In the case of initial data with small total variation, the unform estimate (2.2) on the total variation of the approximate solutions $U_{h}$ has been established by Glimm using certain nonlocal functionals of $U_{h}$ [9]. The Glimm functionals are defined on a family of space-like polygonal arcs called $I$-curves. We recall that an $I$-curve is a connected arc which consists of edges of diamond-shaped domains $\Delta$ and which has the property that the index $m$ of the mesh points $a_{m, n}$ on $I$ increase monotonically with increasing values of $x$. The effect of wave interactions on the 
total variation of the approximate solutions $U_{h}$ can be studied by restricting $U_{h}$ to the family of $I$-curves and then partially ordering the $I$-curves according to the following condition:

$$
J_{2} \geqq J_{1}
$$

if and only if $J_{2}$ lies toward larger time.

The total variation of the restriction $U_{h}(\cdot, J)$ of the approximate solution $U_{h}$ to an $I$-curve $J$ is equivalent to the Glimm functional

$$
L(J)=\Sigma\{|\alpha|: \alpha \operatorname{crosses} J\},
$$

i.e. the sum of the absolute values of the magnitudes of all elementary waves in $U_{h}$ which cross $J$. It is easily verified that

$$
\text { const. } T V U_{h}(\cdot, J) \leqq L(J) \leqq \text { const. } T V U_{h}(\cdot, J) \text {. }
$$

The conservation laws for waves (2.6) were employed in [9] to show that the functional $L(J)$ can increase between two consecutive $I$-curves, $J_{2} \geqq J_{1}$, at most by an amount on the order of the total amount of wave interaction in the enclosed diamond $\Delta$; namely

$$
L\left(J_{2}\right) \leqq L\left(J_{1}\right)+\text { const. } Q(4) .
$$

We recall that a pair of $I$-curves $J_{1}$ and $J_{2}$ are said to be consecutive with respect to a fixed diamond $\Delta$ if they coincide except on the boundary of $\Delta$. In particular, if $J_{2} \geqq J_{1}$ then $J_{2}$ contains the upper sides, $W N$ and $N E$, of $\Delta$ while $J_{1}$ contains the lower sides, $W S$ and $S E$.

While the functional $L(J)$ will generally increase between two consecutive $I$-curves as a consequence of wave interactions, the total potential for wave interaction necessarily decreases as a consequence of the recession of waves after interactions. The total potential for wave interaction is measured by the quadratic Glimm functional

$$
K(J)=\Sigma\{|\alpha||\beta|: \alpha, \beta \text { cross } J \text { and approach }\} .
$$

It follows directly from the conservation laws for waves that

$$
K\left(J_{2}\right) \leqq K\left(J_{1}\right)+\text { const. } L\left(J_{1}\right) Q(\Delta)-Q(\Delta)
$$

if $J_{2} \geqq J_{1}$ are consecutive with respect to $\Delta$. The presence of the term $-Q(\Delta)$ is due to the fact that, by definition, the incoming waves of $\Delta$ contribute to $K\left(J_{1}\right)$ but not to $K\left(J_{2}\right)$. Thus, if the total variation of the restriction of $U_{h}$ to $J_{1}$ is sufficiently small, the total potential for wave interaction decreases by an amount on the order of $Q(\Delta)$ :

$$
K\left(J_{2}\right) \leqq K\left(J_{1}\right)-\text { const. } Q(\Delta) .
$$

It follows from an induction argument [9], using the estimates (2.7) and (2.8), that the functional

$$
F(J)=L(J)+\text { const. } K(J)
$$

is nonincreasing with respect to the family of $I$-curves provided that the initial data has sufficiently small total variation and provided that the constant coefficient of $K$ is chosen sufficient large. 
Since the functional $F$ is equivalent to the total variation norm, i.e.

$$
\text { const. } F(J) \leqq T V U_{h}(\cdot, J) \leqq \text { const. } F(J),
$$

it follows that the total variation of the approximate solutions is uniformly bounded by the total variation of the initial data:

$$
T V_{x} U_{h}(x, t) \leqq \text { const. } T V_{x} U(x, 0) .
$$

The uniform bound (2.9) guarantees the existence of solutions. We note that the proof of estimate (2.9), [9], applies equally well to systems of conservation laws which admit linearly degenerate characteristic fields and thereby establishes existence of solutions to such systems in the case where the initial data have small total variation. Even more generally, in the case of initial data with small total variation, it has been shown [11] that Glimm's method of construction and estimates carry over to systems of conservation laws without convexity or degeneracy assumptions.

From the point of view of the decay of the solution, one of the important corollaries of the decay of the quadratic functional $K(J)$ is the uniform boundedness of the total amount of wave interaction. Rewriting estimate (2.8) in the form

$$
Q(\Delta) \leqq \text { const. }\left\{K\left(J_{1}\right)-K\left(J_{2}\right)\right\}
$$

and summing over all diamonds yields the uniform bound

$$
\sum_{\Delta} Q_{h}(\Delta) \leqq \text { const. }\left\{T V_{x} U(x, 0)\right\}^{2} .
$$

The subscript $h$ is introduced in (2.10) simply to recall the dependence of $Q$ on the approximate solution $U_{h}$.

In this paper we shall assume that the solution $U$ is the limit of a sequence of approximate solutions $U_{h}$ which satisfy approximate conservation laws in which the error term is uniformly summable with respect to $h$, i.e.

where

$$
\varepsilon_{j}(\Delta)=\gamma_{j}(\Delta)+\delta_{j}(\Delta)+\operatorname{error}_{j}(\Delta)
$$

$$
\sum_{\Delta}\left|\operatorname{error}_{j}(\Delta)\right| \leqq \text { const., }
$$

the constant being independent of the approximate solution $U_{h}$. It is a straightforward consequence of (2.11) that the approximate solutions $U_{h}$ satisfy (2.9) and that the total amount of wave interaction is uniformly bounded in $h$ :

$$
\sum_{h} Q_{h}(\Delta) \leqq \text { const. }
$$

Following GLIMM \& LAX [10] we shall now derive approximate conservation laws for waves of a given magnitude. Let $C_{j, h}(\Delta)$ denote the amount of cancellation which occurs between the incoming $j$-waves $\gamma_{j}$ and $\delta_{j}$ in the diamond $\Delta$ :

$$
C_{j, h}(\Delta)=\frac{1}{2}\left\{\left|\gamma_{j}\right|+\left|\delta_{j}\right|-\left|\gamma_{j}+\delta_{j}\right|\right\}
$$


or, equivalently,

$$
C_{j, h}(\Delta)=\left\{\begin{array}{cl}
\min \left(\left|\gamma_{j}\right|,\left|\delta_{j}\right|\right) & \text { if } \operatorname{sgn} \gamma_{j} \neq \operatorname{sgn} \delta_{j} \\
0 & \text { otherwise }
\end{array}\right\} .
$$

With the aid of the cancellation term $C_{j, h}(\Delta)$ the local conservation laws (2.11) can be recast in the form

$$
\begin{aligned}
& L_{j, h}^{+}(\Delta)=E_{j, h}^{+}(\Delta)-C_{j, h}(\Delta)+O\left\{P_{j, h}(\Delta)\right\} \\
& L_{j, h}^{-}(\Delta)=E_{j, h}^{-}(\Delta)+C_{j, h}(\Delta)+O\left\{P_{j, h}(\Delta)\right\} .
\end{aligned}
$$

Here $E_{j, h}^{ \pm}$and $L_{j, h}^{ \pm}$denote the total amount of $j$-wave of $( \pm)$ magnitude which enters and leaves the diamond $\Delta$. The error term $P_{j, h}(\Delta)$ is taken to be nonnegative. It is uniformly summable in the sense that

$$
\sum_{\Delta} P_{j, h}(\Delta) \leqq \text { const., }
$$

where the constant is independent of $h$. Summation of the local conservation laws (2.13) over an arbitrary union $A$ of diamonds yields global laws of the form

$$
\begin{aligned}
& L_{j, h}^{+}(\Lambda)=E_{j, h}^{+}(\Lambda)-C_{j, h}(\Lambda)+O\left\{P_{j, h}(\Lambda)\right\} \\
& L_{j, h}^{-}(\Lambda)=E_{j, h}^{-}(\Lambda)+C_{j, h}(\Lambda)+O\left\{P_{j, h}(\Lambda)\right\}
\end{aligned}
$$

where $C_{j, h}$ and $P_{j, h}$, respectively, denote measures which assign a point mass of magnitude $C_{j, h}(\Delta)$ and $P_{j, h}(\Delta)$ to the center of each diamond $\Delta$.

We observe that the total variation of each of the families of measures $C_{j, h}$ and $P_{j, h}$ is uniformly bounded in $h$ as a consequence of the estimate (2.14). Thus, after passing to a subsequence of approximate solutions, we may assume their convergence in the $w^{*}$ topology for measures. Let

$$
\begin{aligned}
C_{j} & =\lim _{h \rightarrow 0} C_{j, h} \\
P_{j} & =\lim _{h \rightarrow 0} P_{j, h} .
\end{aligned}
$$

The fact that the limits $C_{j}$ and $P_{j}$ are finite measures can be regarded as the statement that the solution $U$ contains a finite amount of wave cancellation and interaction.

For the purpose of studying decay it is also convenient to introduce the measure $Q_{h}$ which assigns a point mass of magnitude $Q_{h}(\Delta)$ to the center of each diamond $\Delta$. Without loss of generality we may also assume that $Q_{h}$ converge in $w^{*}$-topology to a measure which we shall denote by

$$
Q=\lim _{h \rightarrow 0} Q_{h} \text {. }
$$

\section{§ 2. Generalized Characteristics}

The method of GLIMM \& LAX [10] of constructing generalized characteristics for genuinely nonlinear systems of two conservation laws is also applicable to the construction of generalized characteristics for the genuinely nonlinear fields of general systems of $n$ conservation laws. We recall that a generalized $j$-characteristic 
$X_{j}(t)$ exists as the limit of a sequence of approximate characteristics $X_{j, h}(t)$ in the approximate solutions $U_{h}$. The approximate characteristics converge uniformly on bounded intervals of time. Their derivatives converge pointwise with the possible exception of a countable set of values of $t$ :

$$
\dot{X}_{j}(t)=\lim _{j \rightarrow 0} \dot{X}_{j, h}(t)
$$

The one-sided equicontinuity of approximate solutions along approximate characteristics guarantees that the limit $X_{j}(t)$ propagates at the correct speed in the following sense (cf. (5.20)). Let $U^{ \pm}$denote the one-side limits of $U$ along the characteristic $X_{j}$ :

$$
U^{ \pm}=U\left\{X_{j}(t) \pm 0, t\right\}
$$

At points of continuity of the restriction, i.e. $U^{+}=U^{-}$, the characteristic $X_{j}$ propagates at characteristic speed:

$$
\dot{X}_{j}(t)=\lambda_{j}\left\{U\left(X_{j}(t), t\right)\right\} .
$$

At points of discontinuity of the restriction, the characteristic $X_{j}$ propagates at shock speed:

$$
\dot{X}_{j}(t)=\sigma_{j}\left\{U^{+}, U^{-}\right\}
$$

In equation (2.19) the shock speed $\sigma_{j}$ is determined from the Rankine-Hugoniot relations, i.e.

$$
-\sigma_{j}\left[U^{+}-U^{-}\right]+\left[F\left(U^{+}\right)-F\left(U^{-}\right)\right]=0 .
$$

The proofs of the aforementioned results [10] carry over virtually without modification to the genuinely nonlinear fields of a general systems of conservation laws. We do note, however, one minor modification which is needed for the construction of approximate chaacteristics in the more general situation. We recall that in general the diamon-shaped domains $\Delta$ contain two incoming $j$-waves and one outgoing $j$-wave. Thus, in general there exist eight possible configurations of $j$ waves in a typical diamond $\Delta$. However, in the special case of systems of two conservation laws satisfying the Glimm-Lax shock interaction condition [10] only six combinations are permissible: if both of the incoming $j$-waves have magnitudes of the same sign the outgoing $j$-wave will necessarily have a magnitude of that same sign. Only the six dynamically permissible combinations are considered by GLIMM \& LAX in [10]. For general systems it is possible a priori for the interaction terms to dominate in the approximate conservation laws for waves, in the sense that

while

$$
\varepsilon_{j}=\gamma_{j}+\delta_{j}+O\{Q(\Delta)\}
$$

$$
\operatorname{sign} \varepsilon_{j}=-\operatorname{sign} \gamma_{j}=-\operatorname{sign} \delta_{j} .
$$

However, in this situation the outgoing $j$-wave is small in the sense that its magnitude is bounded by the total amount of interaction in $\Delta$ :

$$
\left|\varepsilon_{j}\right| \leqq O\{Q(\Delta)\},
$$


and the continuation program for approximate $j$-characteristics is as follows. If an approximate $j$-characteristic enters a diamond in which both incoming $j$ waves are rarefaction waves while the outgoing $j$-wave is a shock, then the approximate $j$-characteristic is continued (in the only way possible) as the outgoing $j$ shock. If an approximate $j$-characteristic enters a diamond in which both incoming $j$-waves are shocks while the outgoing $j$-wave is a rarefaction wave, the approximate $j$-characteristic is continued as, say, the left edge of the outgoing $j$-rarefaction wave. The choice of either the left or right edge is permitted provided that it is done consistently. Approximate characteristics must be constructed in such a way that any two of the same field do not cross in the $x-t$ plane.

\section{§ 3. Generalized Entropy}

Consider a system of conservation laws of the form

$$
U_{t}+F(U)_{x}=0
$$

A strictly convex function $\eta=\eta(U)$ is called a generalized entropy for system (2.20) with entropy flux $q=q(U)$ provided that all smooth solutions of (2.20) satisfy an additional conservation law of the form

$$
\eta(U)_{t}+q(U)_{x}=0 .
$$

It follows from the quasilinear form of (2.20) and (2.21), i.e.

$$
\begin{aligned}
& U_{t}+F^{\prime}(U) U_{x}=0 \\
& \nabla \eta U_{t}+\nabla q U_{x}=0,
\end{aligned}
$$

that the compatibility condition

$$
\nabla \eta F^{\prime}=\nabla q
$$

is necessary for the existence of the pair $(\eta, q)$. For a broad class of systems of two conservation laws, LAX [16] has established the existence of a solution pair $(\eta, q)$ where $\eta$ is strictly convex. Although the system of equations (2.22) is overdetermined for $n>2$, most of the conservative systems of mathematical physics are endowed with an additional conservation law (2.21) in which $\eta$ is strictly convex. In particular, we mention the following systems: the isentropic equations of gas dynamics in both nonrelativistic and relativistic form [2], [25], the equations of shallow water waves [2], the general equations of gas dynamics in both nonrelativistic and relativistic form [2], [25], the equations of magneto-fluid-dynamics [14] and the equations of finite amplitude plane elastic waves [15]. We also note that an arbitrary symmetric hyperbolic system,

$$
\begin{gathered}
U_{t}+\nabla \phi_{x}=0 \\
\phi=\phi(U): \mathbb{R}^{n} \rightarrow \mathbb{R},
\end{gathered}
$$

possesses a strictly convex generalized entropy

$$
\eta(U)=\frac{1}{2}\|U\|^{2} \text {. }
$$


First, we shall record the aforementioned systems of mathematical physics along with their generalized entropies. For the equations of gas dynamics the density of negative (classical) entropy serves as the function $\eta$. In Lagrange coordinates the nonrelativistic equations of gas dynamics assume the form (2.20) with

$$
U=\left[\begin{array}{l}
\tau \\
u \\
E
\end{array}\right] \text { and } F(U)=\left[\begin{array}{c}
-u \\
p \\
u p
\end{array}\right]
$$

Here $\tau$ denotes the specific volume of the fluid, $u$ the velocity, $p$ the pressure and $E$ the total energy, i.e. the sum of the kinetic energy $\frac{1}{2} u^{2}$ and the specific internal energy $\varepsilon$,

$$
E=\frac{1}{2} u^{2}+\varepsilon .
$$

The additional conservation law (2.21) assumes the form

$$
(-S)_{t}=0,
$$

where $S$ denotes the specific entropy. For a polytropic gas a striaghtforward calculation shows that

$$
-S=-S(\tau, u, E)
$$

is a strictly convex function of the state variables $\tau, u$ and $E$. Specifically,

$$
S(\tau, \varepsilon)=\log \varepsilon+(\gamma-1) \log \tau,
$$

where the adiabatic constant $\gamma$ is greater than one. Therefore,

$$
\eta(U)=-S(\tau, u, E)=-\log \left(E-\frac{1}{2} u^{2}\right)-(\gamma-1) \log \varepsilon .
$$

More generally, it has been shown by FrIEDRICHS \& LAX [8] that the convexity of $-S$ as a function of the state variables $\tau, u$ and $E$ is equivalent to the convexity of total energy $E$ as a function of $\tau, u$ and $S$ :

$$
E=\frac{1}{2} u^{2}+\varepsilon(\tau, S) .
$$

Thus, it is sufficient to postulate the convexity of specific internal energy as a function of $\tau$ and $S$.

The isentropic equations of gas dynamics in Lagrange coordinates can be derived by setting $S$ equal to a constant, say $S_{0}$. The isentropic equations assume the form (2.20) with

$$
U=\left[\begin{array}{l}
\tau \\
u
\end{array}\right] \text { and } F(U)=\left[\begin{array}{c}
-u \\
p
\end{array}\right]
$$

where $p=p\left(\tau, S_{0}\right)$. The energy equation is retained as the additional conservation law:

$$
E\left(\tau, u, S_{0}\right)_{t}+\left(u p\left(\tau, S_{0}\right)\right)_{x}=0 .
$$

In this situation the convexity of $\eta(U)=E\left(\tau, u, S_{0}\right)$ is a direct consequence of the convexity of $E$ as a function of the three arguments $\tau, u$ and $S$. 
In Eulerian coordinates the equations of gas dynamics assume the form (2.20) with

$$
U=\left[\begin{array}{c}
\rho \\
m \\
E
\end{array}\right] \text { and } F(U)=\left[\begin{array}{c}
m \\
m^{2} / \rho+p \\
m E / \rho+m p / \rho
\end{array}\right] .
$$

Here $\rho \equiv 1 / \tau$ denotes the mass per unit volume, $m \equiv \rho u$ the momentum per unit volume and $E$ the total energy per unit volume,

$$
E=\frac{1}{2} \rho u^{2}+\rho \varepsilon .
$$

The additional conservation law assumes the form

$$
(-\rho S)_{t}+(-m S)_{x}=0 \text {. }
$$

In particular, for a polytropic gas

$$
S=\log \left(E-\frac{m^{2}}{2 \rho}\right)-\gamma \log \rho,
$$

and a straightforward calculation shows that

$$
\eta(U) \equiv-\rho S=-\rho \log \left(E-\frac{m^{2}}{2 \rho}\right)-\gamma \rho \log \rho
$$

is a strictly convex function of the state variables $\rho, m$ and $E$. More generally, one may again simply postulate the convexity of

$$
\rho \varepsilon(\rho, S)
$$

as a function of $\rho$ and $S$ or equivalently the convexity of total energy

$$
E=\frac{1}{2} \frac{m^{2}}{\rho}+\rho \varepsilon(\rho, S)
$$

as a function $\rho, m$ and $S$. The isentropic equations in Eulerian coordinates can be derived by setting $S=S_{0}$ in (2.24) and retaining the energy equation as the additional conservation law. The above remarks also apply to the equations of shallow waves [2] since their form is identical to that of the isentropic equations of gas dynamics for a polytropic gas with adiabatic exponent $\gamma=2$.

The relativistic equations of gas dynamics assume the form (2.20) which in component form becomes

$$
\frac{\partial}{\partial t} u_{j}+\frac{\partial}{\partial x} f_{j}=0
$$

where

$$
\begin{aligned}
& u_{1}=\frac{\sigma}{\sqrt{1-u^{2} / c^{2}}} \\
& u_{2}=\left\{\rho+c^{-2}(\rho \varepsilon+p)\right\}\left\{\frac{u}{1-u^{2} / c^{2}}\right\} \\
& u_{3}=\left\{\rho c^{2}+\rho \varepsilon+p\right\}\left\{\frac{c^{2}}{c^{2}-u^{2}}\right\}
\end{aligned}
$$


and

$$
\begin{aligned}
& f_{1}=\frac{u}{\sqrt{1-u^{2} / c^{2}}} \\
& f_{2}=\left\{\rho+c^{2}(\rho \varepsilon+p)\right\}\left\{\frac{u^{2}}{1-u^{2} / c^{2}}\right\}-p \\
& f_{3}=\left\{\rho c^{2}+\rho \varepsilon+p\right\}\left\{\frac{u}{c^{2}-u^{2}}\right\} .
\end{aligned}
$$

Here $\rho$ denotes the rest density, $p$ the pressure, $u$ the velocity and $\varepsilon$ the internal energy per unit rest mass. The additional conservation law assumes the form

$$
\frac{\partial}{\partial t}\left\{\frac{-S}{\sqrt{1-u^{2} / c^{2}}}\right\}+\frac{\partial}{\partial x}\left\{\frac{-\rho u S}{\sqrt{1-u^{2} / c^{2}}}\right\}=0,
$$

where $S$ denotes the specific rest entropy. We refer the reader to TaUB [26] for a derivation of the relativistic equations of gas dynamics and to FRIEDRICHS [7], Sections 5 and 7 , for a verification of the convexity condition. In particular, it is shown in [7], Section 7, that if the internal energy per unit rest volume,

$$
\rho \varepsilon(\rho, S)
$$

is a convex function of $\rho$ and $S$ then the generalized entropy

$$
\eta \equiv \frac{-\rho S}{\sqrt{1-u^{2} / c^{2}}}
$$

becomes a convex function when expressed in terms of the variables $u_{j}$. We refer the reader to [7], Section 3, for a more general formulation of the convexity which is invariant under arbitrary coordinates changes of the dependent variables. The relativistic equations of isentropic gas dynamics can be derived in the same manner as in the non-relativistic case.

The Lundquist equations of magneto-fluid-dynamics in Lagrange coordinates assume the form (2.20) with

$$
U=\left[\begin{array}{c}
u \\
v \\
w \\
E \\
\tilde{H}_{2} \\
\tilde{H}_{3}
\end{array}\right] \text { and }-F(U)=\left[\begin{array}{c}
u \\
p^{*} \\
\mu H_{1} H_{2} / 4 \pi \\
\mu H_{1} H_{3} / 4 \pi \\
-u p+v \cdot H \\
H_{1} v \\
H_{1} w
\end{array}\right]
$$

Here $v=(u, v, w)$ denotes the fluid velocity vector, $H=\left(H_{1}, H_{2}, H_{3}\right)$ the magnetic field vector, $p^{*}$ the total pressure, i.e. the sum of the fluid pressure $p$ and the magnetic pressure $p_{m}$,

$$
\begin{gathered}
p^{*}=p+p_{m} \\
p_{m} \equiv \frac{\mu}{8 \pi}\|H\|^{2}=\frac{\mu}{8 \pi}\left\{H_{1}^{2}+H_{2}^{2}+H_{3}^{2}\right\}
\end{gathered}
$$


$E$ the total energy,

and

$$
E=\frac{1}{2}\|v\|^{2}+\varepsilon+\tau p_{m}
$$

$$
\begin{aligned}
& \tilde{H}_{2}=\tau H_{2} \\
& \tilde{H}_{3}=\tau H_{3}
\end{aligned}
$$

where $\tau$ denotes the specific volume. We note that the $x$-component of the magnetic field vector, $H_{1}$, is constant. As in the case of gas dynamics the additional conservation law assumes the form

For a polytropic gas

$$
(-S)_{t}=0
$$

$$
-S=-\log \varepsilon+\log \tau^{\gamma-1}
$$

and the generalized entropy takes the form

$$
\eta(U) \equiv-S=-\log \left\{E-\frac{1}{2}\|v\|^{2}-\frac{\mu}{8 \pi}\|H\|^{2}\right\}-(\gamma-1) \log \tau .
$$

In analogy with gas dynamics, one may simply postulate the convexity of internal energy $\varepsilon$ as a function of $\tau$ and $S$ or, equivalently, the convexity of total energy

$$
E=\frac{1}{2}\|v\|^{2}+\varepsilon(\tau, S)+\tau p_{m}
$$

as a function of the state variables $\tau, u, v, w, \tilde{H}_{2}, \tilde{H}_{3}$ and $S$. The situation in Eulerian coordinates is similar.

Lastly we recall the structure of the equations of finite amplitude plane elastic waves (cf. [15], Section 4). These equations assume the form (2.20) with

$$
U=\left[\begin{array}{l}
u \\
v
\end{array}\right] \quad \text { and } \quad F(U)=\left[\begin{array}{c}
\nabla \phi(v) \\
-u
\end{array}\right]
$$

where

$$
u=\left[\begin{array}{l}
u_{1} \\
u_{2} \\
u_{3}
\end{array}\right] \text { and } v=\left[\begin{array}{l}
v_{1} \\
v_{2} \\
v_{3}
\end{array}\right]
$$

Here we consider a hyperelastic material with strain energy function $\phi=$ $\phi\left(v_{1}, v_{2}, v_{3}\right)$. The additional conservation law expresses the conservation of total mechanical energy:

$$
\left\{\frac{1}{2}\left(u_{1}^{2}+u_{2}^{2}+u_{3}^{2}\right)+\phi(v)\right\}_{t}+\{u \cdot \nabla \phi\}_{x}=0
$$

A straightforward calculation shows that the strict convexity of $\phi$ is guaranteed by the strict hyperbolicity of the system (2.25).

Next, we recall certain basic facts concerning the role of generalized entropy in the admissibility of solutions. These will be used later in this subsection to derive one of the main estimates which we need in Section 3 to establish the decay of shock waves. As noted already, we shall consider solutions $U$ constructed by the Glimm difference scheme. It is known [18] that such solutions satisfy the entropy inequality of LAX, i.e.

$$
\frac{\partial}{\partial t} \eta(U)+\frac{\partial}{\partial x} q(U) \leqq 0
$$


in the sense of distributions. The entropy inequality (2.26) can also be expressed in an equivalent pointwise form [18]. Consider, for simplicity, a solution $U$ of a Riemann problem which consists precisely of two constant states $U_{l}$ and $U_{r}$ connected by a line of discontinuity $x / t=s$. It can be shown in a straightforward way that $U$ satisfies the entropy inequality (2.26) if and only if

$$
s[\eta]-[q] \leqq 0
$$

Here the square bracket denotes the jump in the enclosed quantity from right to left across the discontinuity, e.g.

$$
[\eta]=\eta\left(U_{l}\right)-\eta\left(U_{r}\right)
$$

The pointwise inequality (2.27) can be derived in a manner similar to that of the Rankine-Hugoniot relations

$$
s[U]-[F]=0,
$$

(cf. [19]). The equivalence of (2.26) and (2.27) can also be established within the class of piecewise smooth solutions. In the latter context the speed of propagation $s$ of a shock wave $x=x(t)$ is given by

$$
s=\frac{d x}{d t}
$$

and the corresponding jumps in $\eta$ and $q$ by

$$
\begin{aligned}
& {[\eta]=\eta\{U(x(t)-0, t)\}-\eta\{U(x(t)+0, t)\}} \\
& {[q]=q\{U(x(t)-0, t)\}-\eta\{U(x(t)+0, t)\} .}
\end{aligned}
$$

For our present purposes it is not necessary to provide a pointwise interpretation of the entropy inequality (2.26) in a setting more general than that of piecewise smooth solutions. A pointwise interpretation of (2.26) for functions of bounded variation is given in Section 5, Proposition 5.2.

The entropy inequality has a simple physical interpretation in the case of the equations of gas dynamics in both nonrelativistic and relativistic form and in the case of the equations of magneto-fluid-dynamics. For a shock wave, condition (2.27) is equivalent to the condition that classical specific entropy increase from front to back across the shock, [2], [14], [26]. The conditions under which this equivalence has been established include the case of a polytropic gas. In general, it has been shown by LAX [18] that strict inequality holds in (2.27) for sufficiently weak shocks and furthermore that the quantity $s[\eta]-[q]$ is third order in the magnitude $\varepsilon$ of the shock:

$$
s[\eta]-[q]=O\left(\varepsilon^{3}\right) .
$$

The third order property (2.28) generalizes the classical result in gas dynamics that the change in (classical) specific entropy is third order in the magnitude of the shock.

In contrast to discontinuities corresponding to genuinely nonlinear fields, all generalized contact discontinuities are admissible. If $U_{l}$ and $U_{r}$ are connected by a generalized contact discontinuity then

$$
s[\eta]-[q]=0 .
$$


The equality (2.29) can be established by considering the restriction of the left hand side of (2.29) to a $j$-wave curve corresponding to a linearly degenerate field, i.e.

$$
s\left\{\eta\left(U_{j}(\varepsilon)\right)-\eta\left(U_{j}(0)\right)\right\}-\left\{q\left(U_{j}(\varepsilon)\right)-q\left(U_{j}(0)\right)\right\}=0,
$$

and differentiating with respect to $\varepsilon$. The result of this operation together with the compatibility condition (2.22) yields (2.29).

In this paper we shall assume for simplicity that strict inequality holds in (2.27) for $j$-shocks of arbitrary magnitude. As we remarked above this condition is satisfied by the equations of fluid flow since the specific entropy necessarily increases from the front to the back of the shock. In general this assumption guarantees an estimate of the form

$$
s[\eta]-[q] \leqq- \text { const. }|\varepsilon|^{3},
$$

where the constant is positive and uniformly bounded away from zero provided that the states $U_{l}$ and $U_{r}$ on the left and right sides of the shock lie in a given compact set. We note that in the case of equations of fluid dynamics the latter restriction implies that the density of the fluid on both sides of the shock is strictly positive. Depending on the choice of wave magnitude, estimate (2.30) may or may not be uniform at arbitrarily low densities.

One of the main consequences of the entropy inequality (2.26) is the boundedness of total entropy. Fix a constant $\bar{U}$. It has been observed by Dafermos [3] that

$$
\bar{\eta}(U)=\eta(U)-\eta(\bar{U})-\sum_{i=1}^{n} \frac{\partial}{\partial u_{i}} \eta(\bar{U})\left\{u_{i}-\bar{u}_{i}\right\}
$$

is a positive strictly convex generalized entropy, i.e.

$$
\bar{\eta}(U)>0 \quad \text { if } U \neq \bar{U} .
$$

The associated entropy flux is given by

$$
\bar{q}(U)=q(U)-q(\bar{U})-\sum_{i=1}^{n} \frac{\partial}{\partial u_{i}} \eta(\bar{U})\left\{f_{i}(U)-f_{i}(\bar{U})\right\} .
$$

Consider a solution $U$ whose initial data $U(x, 0)$ are equal to a constant $\bar{U}$ outside a bounded interval. It follows from a result of DAFERMOs [4] that the rate of decay of total entropy at a given time $t$ is proportional to the sum of the cubes of the magnitudes of all shocks which exist in the solution at time $t$. Expressed in terms of the pair $(\bar{\eta}, \bar{q})$ this result reads

$$
\frac{d}{d t} \int_{-\infty}^{\infty} \bar{\eta}\{U(x, t)\} d x=\sum_{\text {shocks }}\{s[\bar{\eta}]-[\bar{q}]\}(t) .
$$

Integrating (2.31) from zero to $T$ yields

$$
\int_{-\infty}^{\infty} \bar{\eta}\{U(x, t)\} d x-\int_{0}^{T} \sum_{\text {shocks }}\{+s[\bar{\eta}]-[\bar{q}]\} d t=\int_{-\infty}^{\infty} \bar{\eta}\{U(x, 0)\} d x .
$$


It follows from (2.32) that the total entropy of the solution, as measured by $\bar{\eta}$, is uniformly bounded in time,

$$
\int_{-\infty}^{\infty} \bar{\eta}\{U(x, t)\} d x \leqq \text { const. }
$$

and therefore that the total entropy of the solution, as measured by an arbitrary convex entropy $\eta$, is uniformly bounded in time:

$$
\left|\int_{-\infty}^{\infty}[\eta(U(x, t))-\eta(\bar{U})] d x\right| \leqq \text { const. }
$$

Furthermore, it follows from the third order property (2.28) that

$$
\int_{0}^{T} \sum_{\text {shocks }}|[U]|^{3}(t) d t \leqq \text { const. }
$$

The estimates (2.32)-(2.35) can also be established for admissible solutions which are only assumed to be functions of bounded variation in the sense of CESARI [26]. However, in this paper we shall only need these estimates in the case of piecewise smooth functions.

\section{Decay of Shock Waves}

In this section we shall restrict our attention to systems of conservation laws which possess a strictly convex entropy. Consider a sequence of approximate solutions $U_{h}$ and let $\Omega(t)$ denote the set of magnitudes of those shock waves in $U_{h}$ which intersect $\{(x, \tau): \tau=t\}$. Let

$$
V_{h}(t)=\Sigma\left\{|\delta|^{3}: \delta \in \Omega(t)\right\}
$$

The total cubic variation of shock waves dominates the maximum norm of shock waves, i.e.

in the sense that

$$
M_{h}(t)=\max \{|\delta|: \delta \in \Omega(t)\}
$$

$$
M_{h}^{3}(t) \leqq V_{h}(t) .
$$

The uniform decay of shock waves in a sequence of approximate solutions is a consequence of two facts: the uniform boundedness of total entropy and the uniform boundedness of total wave interaction. The former bound implies that the function $V_{h}$ is "nearly" integrable and the latter bound that $V_{h}$ is "nearly" uniformly continuous.

Let

$$
\begin{gathered}
V_{h}\left[t_{1}, t_{2}\right]=\sup \left\{V_{h}(t): t_{1} \leqq t \leqq t_{2}\right\} \\
M_{h}\left[t_{1}, t_{2}\right]=\sup \left\{M_{h}(t): t_{1} \leqq t \leqq t_{2}\right\}
\end{gathered}
$$

Lemma 3.1. For every $\varepsilon>0$ there exists a constant $T=T(\varepsilon)$ such that for each fixed $S>0$ we have

$$
\varlimsup_{h \rightarrow 0} V_{h}[T, T+S] \leqq \varepsilon .
$$


It is an immediate corollary of (3.2) that the maximum limiting strength of shock waves contained in bounded intervals of time approaches zero with large time uniformly in the length of the interval:

Thus, in particular

$$
\varlimsup_{h \rightarrow \infty} M_{h}[T, T+S] \leqq \varepsilon^{1 / 3} .
$$

$$
\lim _{t \rightarrow \infty} \varlimsup_{h \rightarrow 0} M_{h}(t)=0 .
$$

Proof of Lemma 3.1. Fix an approximate solution $U_{h}$ and consider two $I$ curves $J_{2} \geqq J_{1}$ which are consecutive with respect to a diamond $\Delta$. The conservation laws for waves (2.13) imply that the strength of the outgoing $j$-shock is less than or equal to the sum of the strengths of the incoming $j$-shocks plus an error term

$$
\left|\varepsilon_{j}^{-}\right| \leqq\left|\gamma_{j}^{-}\right|+\left|\delta_{j}^{-}\right|+P_{j, h}(\Delta) .
$$

Thus the cubic magnitudes satisfy

$$
\left|\varepsilon_{j}^{-}\right|^{3} \leqq\left|\gamma_{j}^{-}\right|^{3}+\left|\delta_{j}^{-}\right|^{3}+\text { const. }\left\{P_{j, h}(\Delta)+Q_{h}(\Delta)\right\} .
$$

Therefore the cubic variation $V_{h}(J)$ of the restriction of the approximate solution $V_{h}$ to the $I$-curve $J$ satisfies

where

$$
V_{h}\left(J_{2}\right) \leqq V_{h}\left(J_{1}\right)+\mu_{j, h}(\Delta)
$$

$$
\mu_{j, h}(\Delta) \stackrel{\text { def }}{=} \text { const. }\left\{P_{j, h}(\Delta)+Q_{h}(\Delta)\right\} .
$$

Summing over all diamonds $\Delta$ in the strip

we obtain

$$
\mathscr{S}\left[t_{1}, t_{2}\right]=\left\{(x, t): t_{1} \leqq t \leqq t_{2}\right\},
$$

$$
V_{h}\left(t_{2}\right) \leqq V_{h}\left(t_{1}\right)+\mu_{j, h}\left(\mathscr{P}\left[t_{1}, t_{2}\right]\right) .
$$

For simplicity in writing we shall denote the strip $\mathscr{S}$ by $\left[t_{1}, t_{2}\right]$.

Applying estimate (2.32) to the approximate solution $U_{h}$ yields

$$
\int_{0}^{T} V_{h}(t) d t \leqq \text { const. + const. } \sum_{n \leq T}\left|\int_{-\infty}^{\infty} \eta\left\{U_{h}(x, n s)\right\}-\eta\left\{U_{h}(x, n s-0)\right\} d x\right| .
$$

The contributions from the interfaces between the layers at times $t=n s$ are uniformly of order $h$ (cf. [9]), thus

$$
\left|\int_{-\infty}^{\infty}\left[\eta\left\{U_{h}(x, n s)\right\}-\eta\left\{U_{h}(x, n s-0)\right\}\right] d x\right| \leqq O(h) T V U(x, 0) .
$$

Therefore,

$$
\int_{0}^{h^{-1}} V_{h}(t) d t \leqq c_{1}
$$

where the constant $c_{1}$ is independent of $h$.

Fix $\varepsilon$ and $S$. Let $\mu$ denote the $w^{*}$-limit of the sequence of measures $\mu_{h}$. We recall that both $P_{j, h}$ and $Q_{h}$ converge in the $w^{*}$-topology. Choose a constant $c_{2}$ 
such that

$$
\mu_{h}\{[0, \infty)\}<c_{2}
$$

and let $c_{3}=c_{1}+c_{2}$. Choose a constant $\tau>1$ so large that

and define $T=T(\varepsilon)$ by

$$
\mu\{[\tau, \infty)\}<\varepsilon
$$

$$
T(\varepsilon)=c_{3} \tau / \varepsilon .
$$

It follows from the $w^{*}$-convergence of the measures $\mu_{h}$ that there exists a constant $H_{1}=H_{1}(\varepsilon, S)$ such that

if $h \leqq H_{1}$. Let

$$
\mu_{h}\{[\tau, T+S]\}<2 \varepsilon
$$

$$
H(\varepsilon, S)=\min \left\{H_{1},(T+S)^{-1}\right\} .
$$

Integration of inequality (3.4) with respect to $t$, from zero to a fixed value $t>\tau$ yields

$$
V_{h}(t) \leqq \frac{1}{t} \int_{0}^{t} V_{h}(s) d s+\frac{1}{t} \int_{0}^{\tau} \mu_{h}\{[s, t]\} d s+\frac{1}{t} \int_{\tau}^{t} \mu_{h}\{[s, t]\} d s .
$$

Consider values of $t$ and $h$ such that $T(\varepsilon) \leqq t \leqq T(\varepsilon)+S$ and $h \leqq H$. The first term in (3.5) is less than $c_{1} / t$, which in turn satisfies

$$
c_{1} / t \leqq c_{1} / T \leqq c_{1} \varepsilon / c_{3} \tau<\varepsilon
$$

Since the total variation of $\mu_{h}$ is bounded by $c_{2}$ the second term in (3.5) is less than

$$
\tau c_{2} / t \leqq \tau c_{2} / T<\varepsilon .
$$

The third term is also less than $2 \varepsilon$ since the integrand satisfies

We conclude that

$$
\mu_{h}\{[s, t]\} \leqq \mu_{h}\{[\tau, T+S]\} \leqq 2 \varepsilon
$$

$$
V_{h}(t) \leqq 4 \varepsilon
$$

if $h \leqq H(\varepsilon, S)$. This completes the proof of the lemma.

\section{Decay of the Oscillation}

Consider a sequence of approximate solutions $U_{h}$. Fix an index $j$ and let $\Omega_{j, h}(a, b, \tau)$ denote the set of magnitudes of those $j$-waves in $U_{h}$ which intersect

$$
\{(x, t): a \leqq x<b, t=\tau\} .
$$

The large-time asymptotic behavior of waves of a fixed characteristic field can be conveniently studied in terms of the following measures:

$$
\begin{aligned}
\omega_{j, h}^{+}[a, b) & =\Sigma\left\{\delta: \delta \in \Omega_{j, h}, \delta>0\right\} \\
\omega_{j, h}^{-}[a, b) & =\Sigma\left\{|\delta|: \delta \in \Omega_{j, h}, \delta<0\right\} \\
\omega_{j, h} & =\omega_{j, h}^{+}+\omega_{j, h}^{-} \\
\theta_{j, h}[a, b) & =\Sigma\left\{|\delta|^{3}: \delta \in \Omega_{j, h}, \delta<0\right\} .
\end{aligned}
$$


The dependence on $\tau$ has been suppressed for simplicity in printing. Since the total variation of the measures (4.1) is uniformly bounded in $h$ there exists a subsequence of approximate solutions such that the following limits exist at all rational times $\tau$ in the $w^{*}$-topology for measures:

$$
\begin{aligned}
\omega_{j}^{+} & =\lim _{h \rightarrow 0} \omega_{j, h}^{+} & \omega_{j}^{-} & =\lim _{h \rightarrow 0} \omega_{j, h}^{-} \\
\omega_{j} & =\lim _{h \rightarrow 0} \omega_{j, h} & \theta_{j} & =\lim _{h \rightarrow 0} \theta_{j, h} .
\end{aligned}
$$

We shall say that the eigenvalues $\lambda_{j}$ are strictly separated on the range of the approximate solutions $U_{h}$ if there exists a compact set $B$ such that

and such that

$$
B \supset\left\{U_{h}(x, t): h>0,-\infty<x<\infty, t>0\right\}
$$

$$
\sup \left\{\lambda_{j-1}(U): U \in B\right\}<\inf \left\{\lambda_{j}(U): U \in B\right\} .
$$

As we remarked in the introduction the eigenvalues of the equations of gas dynamics in Lagrange coordinates are strictly separated on an arbitrary compact set. In general, the strict separation of the eigenvalues together with the decay of shock waves as expressed by (3.3) implies that the speed of propagation of the elementary waves of distinct characteristic fields are strictly separated at large times. More precisely, let $\alpha_{j, h}(t)$ and $\beta_{j, h}(t)$ denote the minimum and maximum speed of propagation of $j$-waves in $U_{h}$ at time $t$. Choose $\delta>0$ along with non-zero constants $\mu_{j}$ such that

$$
\mu_{j-1}+\delta<\inf \left\{\lambda_{j}(U): U \in B\right\}<\sup \left\{\lambda_{j}(\mathrm{U}): U \in B\right\}<\mu_{j}-\delta .
$$

It follows from Lemma 3.1 that there exists a time $T$ such that

$$
\mu_{j-1}+\delta \leqq \varliminf \alpha_{j, h}(t) \leqq \varlimsup \lim \beta_{j, h}(t) \leqq \mu_{j}-\delta
$$

if $t \geqq T$. Furthermore the limits in (4.2) are taken on uniformly in $h$ if $t$ lies in bounded intervals of time.

The following lemma may be regarded as the statement that the total variation of the solution restricted to the cone

$$
K_{j}=\left\{(x, t): \mu_{j-1}<x / t<\mu_{j}\right\}
$$

is asymptotically supported by $j$-waves only. The decay of $k$-waves, $k \neq j$, is a consequence of the conservation laws for waves (2.13) and the strict separation of the eigenvalues. Let $K_{j}(t)$ denote the cross-section

$$
K_{j}(t)=K_{j} \cap\{(x, \tau): \tau=t\} .
$$

Lemma 4.1. Suppose that the eigenvalues are strictly separated. Then for each index $j$

$$
\lim _{t \rightarrow \infty} \sum_{k \neq j} \omega_{k}\left\{K_{j}(t)\right\}=0,
$$

where the limit is taken through rational values of $t$. 
It is an immediate corollary of Lemma 4.1 that the same sequence of approximate solutions satisfies

for all values of $t$.

$$
\lim _{t \rightarrow \infty} \varlimsup_{h \rightarrow 0} \sum_{k \neq j} \omega_{k, h}\left\{K_{j}(t)\right\}=0
$$

Sketch of proof of Lemma 4.1. Fix an index $j$. For concreteness we shall consider waves of the $(j+1)^{\text {st }}$ characteristic field and show that

$$
\lim _{t \rightarrow \infty} \omega_{j+1}\left\{K_{j}(t)\right\}=0 .
$$

Without loss of generality we may assume that $\mu_{j-1}>0$. Let $l_{1}$ and $l_{2}$ denote straight lines which pass through the left and right end points of the crosssection $K_{j}(t)$ and which propagate at the speeds $\mu_{j+2}$ and $\mu_{j+1}+\delta / 2$, respectively. If the support of the initial data is contained in an interval of the form $[-N, N]$ then the support of all of the Glimm difference approximations is contained in a region $\Gamma$ of the form

$$
\Gamma=\{(x, t):|x| \leqq N+M t, t \geqq 0\} .
$$

Since the $x$-coordinates of the end points of $K_{j}(t)$ are given by

$$
\mu_{j-1} t \text { and } \mu_{j} t
$$

there exists a time $\tau=O(t)$ such that the $x$-interval $I(\tau)$ which lies between $l_{1}$ and $l_{2}$ at time $\tau$ is contained in the complement of $\Gamma$.

Consider the quadrilateral region $R=R(t)$ bounded by the segments $K_{j}(t)$ and $I(\tau)$ and by the restrictions of the lines $l_{1}$ and $l_{2}$ to the interval $[\tau, t]$. In the limit as $h$ approaches zero the speed of propagation of all elementary $(j+1)$-waves is strictly less than the speed of propagation of the left edge $l_{1}$ of the region $R$ and is strictly greater than the speed of propagation of the right edge $l_{2}$. Thus, in the limit the total strength of all $(j+1)$-waves entering the region $R$ equals zero while the total strength of all $(j+1)$-waves leaving the region equals

$$
\omega_{j+1}\left\{K_{j}(t)\right\} .
$$

It follows from the conservation laws for waves that

$$
\omega_{j+1}\left\{K_{j}(t)\right\} \leqq P_{j}\{R(t)\} .
$$

Since the measure $P_{j}$ is finite the limit of the right hand side of (4.3) approaches zero as $t$ approaches infinity (through rational values). This completes the proof of the lemma.

The oscillation of the solution in cones $K_{j}$ corresponding to the genuinely nonlinear fields is essentially determined by the balance between $j$-shock waves and $j$-rarefaction waves. At large times the influence of $k$-waves, $k \neq j$, is arbitrarily small due to the asymptotic uncoupling of the cahracteristic fields. The quantity

$$
\omega^{j}(t)=\sup \left\{\sum_{k \neq j} \omega_{k}\left\{K_{j}(\tau)\right\}: \tau \geqq t\right\}
$$

serves as an upper bound for the total influence of $k$-waves, $k \neq j$, in the truncated cone

$$
K_{j}[t, \infty)=K_{j} \cap\{(x, \tau): \tau \geqq t\} .
$$


We shall show that in each fixed subinterval of $K_{j}(t)$ the total amount of $j$-shock wave and $j$-compression wave may not exceed the total amount of $j$-rarefaction wave by more than the sum of the following four quantities: $\omega^{j}(t)$; the total amount of wave interaction and cancellation in the truncated cone $K_{j}[t, \infty)$, i.e.

$$
P_{j}(t)=P\left\{K_{j}[t, \infty)\right\} \quad \text { and } \quad C_{j}(t)=C\left\{K_{j}[t, \infty)\right\}
$$

and the maximum limiting strength of shock waves in $K_{j}[t, \infty)$, i.e.

$$
M_{j}(t)=\sup \left\{\varlimsup_{h \rightarrow 0} M_{j, h}(\tau): \tau \geqq t\right\},
$$

where $M_{j, h}(\tau)$ denotes the maximum strength of those shock waves in $U_{h}$ which intersect $K_{j}(\tau)$.

Consider a solution $U$ which exists as the limit of a sequence of Glimm difference approximations. In the following lemma we do not assume the strict separation of the eigenvalues.

Lemma 4.2. Let $j$ denote the index of a genuinely nonlinear field. Suppose that $(x, t)$ and $(y, t)$ lie in the cross-section $K_{j}(t)$ and that $x<y$. Then

$$
\lambda_{j}\{U(x, t)\}-\lambda_{j}\{U(y, t)\} \leqq \text { const. }\left\{P_{j}(t)+C_{j}(t)+M_{j}(t)+\omega^{j}(t)\right\},
$$

where the constant depends only on the $L^{\infty}$-norm of $U$.

It is convenient from the point of view of decay of solutions to measure the magnitudes of the elementary waves of a genuinely nonlinear field by the corresponding eigenvalue. In this case we shall denote the measures $\omega_{j, h}^{ \pm}$and their $w^{*}$-limits $\omega_{j}^{ \pm}$by $\lambda_{j, h}^{ \pm}$and $\lambda_{j}^{ \pm}$respectively. With the aid of the measures $\lambda_{j}^{ \pm}$the main idea in the proof of Lemma 4.2 can be expressed as follows. We may assume without loss of generality that the approximate solutions $U_{h}$ converge pointwise in $x$ for each rational $t$. Therefore, for each rational $t$

$$
\lambda_{j}\{U(x, t)\}-\lambda_{j}\{U(y, t)\}=\lambda_{j}^{-}[x, y]-\lambda_{j}^{+}[x, y]
$$

with the possible exception of countably many points $x$ and $y$ at which $\lambda_{j}^{ \pm}$admit point masses. Now, if the total amount of $j$-shock and compression wave as measured by $\lambda_{j}$, i.e. $\lambda_{j}^{-}[x, y]$, exceeds the total amount of $j$-rarefaction wave, i.e. $\lambda_{j}^{+}[x, y]$, by more than

$$
\text { const. }\left\{P_{j}(t)+C_{j}(t)+M_{j}(t)+\omega^{j}(t)\right\}
$$

then the $j$-characteristics passing through $(x, t)$ and $(y, t)$ will coalesce in a finite time and produce a $j$-shock wave. This phenomenon is similar to the breaking of a compression wave and is a consequence of the condition of genuine nonlinearity. The conservation laws for waves then imply that $\lambda_{j}^{-}[x, y]$ is bounded by the strength of the $j$-shock so produced plus the total amount of wave interaction and cancellation which occurs in the region bounded by the characteristics issuing from $(x, t)$ and $(y, t)$. In general the geometry of $j$-characteristics is influenced by wave interactions; it is for this reason that the term $\omega^{j}(t)$ enters the estimate (4.4). 
Proof of Lemma 4.2. It is sufficient to prove (4.4) for rational values of $t$ since the solution is $L^{1}$-continuous in time [9]:

$$
\int_{-\infty}^{\infty}\left|U\left(x, t_{1}\right)-U\left(x, t_{2}\right)\right| d x \leqq \text { const. }\left|t_{1}-t_{2}\right| .
$$

Fix $(x, \tau)$ and $(y, \tau)$ and let $X_{h}$ and $Y_{h}$ denote the approximate characteristics in $U_{h}$ which pass through these points. We shall define the total amount of $k$-wave between $X_{h}$ and $Y_{h}$ at time $t$ by $\mu_{k, h}(t)$ :

Let

$$
\begin{aligned}
& \mu_{k, h}(t)=\omega_{k, h}\left\{\left[X_{h}(t), Y_{h}(t)\right]\right\}, k \neq j \\
& \mu_{j, h}^{ \pm}(t)=\lambda_{j, h}^{ \pm}\left\{\left[X_{h}(t), Y_{h}(t)\right]\right\}
\end{aligned}
$$

$$
\text { str } X_{h}(t) \text { and } \operatorname{str} Y_{h}(t)
$$

denote the strengths of the approximate characteristics $X_{h}$ and $Y_{h}$ at time $t$. We recall that the strength of a segment of an approximate characteristic is defined to be zero unless the segment is a shock wave in which case the strength is defined to be the absolute value of the magnitude of the shock. After passing to a subsequence of approximate solutions, the limits

$$
\begin{aligned}
X(t) & =\lim X_{h}(t), & Y(t) & =\lim Y_{h}(t) \\
\operatorname{str} X(t) & =\lim \operatorname{str} X_{h}(t), & \operatorname{str} Y(t) & =\lim \operatorname{str} Y_{h}(t) \\
\mu_{k}(t) & =\lim \mu_{k, h}(t), & \mu_{j}^{ \pm}(t) & =\lim \mu_{j, h}^{ \pm}(t)
\end{aligned}
$$

exist with the possible exception of countably many values of $t$ [10]. All of the limits are functions of bounded variation. The convergence of the approximate characteristics $X_{h}$ and $Y_{h}$ is uniform on bounded intervals of time and the limiting characteristics $X$ and $Y$ are Lipschitz continuous.

We shall estimate the rate of convergence of $X$ and $Y$ in terms of the above quantities. The propagation speed of a $j$-shock differs from the value of $\lambda_{j}$ on either side by a quantity of the order of the shock strength. Hence, we have

$$
\dot{X}_{h}(t)=\lambda_{j}\left\{U_{h}\left(X_{h}(t) \pm 0, t\right)\right\} \pm O(1) \operatorname{str} X_{h}(t)
$$

and a similar expression for $\dot{Y}_{h}(t)$. Subtracting and passing to the limit yields

$$
\dot{Y}(t)-\dot{X}(t)=\mu_{j}^{ \pm}(t)-\mu_{j}^{-}(t)+O(1)\{\operatorname{str} X(t)+\operatorname{str} Y(t)\}+O(1) \sum_{k \neq j} \mu_{k}(t) .
$$

The left hand side of (4.7) can be estimated as follows. Consider the region

$$
R[\tau, t]=\{(x, s): X(s) \leqq x \leqq Y(s), \tau \leqq s \leqq t\} .
$$

It follows from the conservation laws for waves that the balance of $j$-waves between $X$ and $Y$ at time $\tau$ and $t$ differ by no more that the total amount of wave interaction in the region $R[\tau, t]$ plus the total amount of cancellation on the lateral boundaries. Therefore,

$$
\mu_{j}^{-}(t)-\mu_{j}^{+}(t) \geqq \mu_{j}^{-}(\tau)-\mu_{j}^{+}(\tau)-P\{R[\tau, t]\}-C_{j}\{X[\tau, t]\}-C_{j}\{Y[\tau, t]\}
$$


The cancellation of $j$-waves in the interior of $R$ occurs (by definition) simultaneously between $j$-shocks and $j$-rarefaction waves and thus does not affect the balance of $j$-waves. However $j$-shock waves which lie on the lateral boundaries

$$
\begin{aligned}
X[\tau, t] & =\{(X(s), s): \tau \leqq s \leqq t\} \\
Y[\tau, t] & =\{(Y(s), s): \tau \leqq s \leqq t\} .
\end{aligned}
$$

can be cancelled by interactions with $j$-rarefaction waves which lie in the complement of $R$. The latter effect is reflected in the fourth and fifth term of (4.8).

With the possible exception of countably many values of $x$ and $y$ we have

$$
\lambda_{j}\{U(x, \tau)\}-\lambda_{j}\{U(y, \tau)\}=\mu_{j}^{-}(\tau)-\mu_{j}^{+}(\tau)+O(1) \sum_{k \neq j} \mu_{k}(\tau) .
$$

Using (4.8) and (4.9), we obtain from (4.7) that

$$
\begin{aligned}
\dot{Y}(t)-\dot{X}(t) \leqq & \lambda_{j}\{U(y, \tau)\}-\lambda_{j}\{U(x, \tau)\}+C_{j}\{X[\tau, t]\} \\
& +C_{j}\{Y[\tau, t]\}+P\{R[\tau, t]\} \\
& +O(1) \sum_{k \neq j} \mu_{k}(t)+O(1)\{\operatorname{str} X(t)+\operatorname{str} Y(t)\} .
\end{aligned}
$$

It follows from (4.10) that

where

$$
Y(t)-\dot{X}(t) \leqq \lambda_{j}\{U(y, \tau)\}-\lambda_{j}\{U(x, \tau)\}+c H(\tau),
$$

$$
H(\tau) \stackrel{\text { def }}{=} P_{j}(\tau)+C_{j}(\tau)+M_{j}(\tau)+\omega^{j}(\tau)
$$

and where $c$ is a constant depending on the $L^{\infty}$-norm of the solution.

In order to establish the estimate (4.4) we need only consider the case where

$$
H(\tau) \leqq \frac{-1}{2 c}\left\{\lambda_{j}(U(y, \tau))-\lambda_{j}(U(x, \tau))\right\},
$$

or equivalently the case where

$$
\dot{Y}(t)-\dot{X}(t) \leqq \frac{1}{2}\left\{\lambda_{j}(U(y, \tau))-\lambda_{j}(U(x, \tau))\right\}, \quad t>\tau .
$$

Furthermore we may assume that the right-hand side of (4.11) is negative. Under these circumstances the characteristics $X$ and $Y$ coalesce in a finite time and it follows from the conservation laws for waves that

$$
\mu_{j}^{-}(\tau)-\mu_{j}^{+}(\tau) \leqq \text { const. }\left\{P_{j}(\tau)+C_{j}(\tau)+M_{j}(\tau)\right\} .
$$

This inequality together with (4.9) yields

$$
\lambda_{j}\{U(x, \tau)\}-\lambda_{j}\{U(y, \tau)\} \leqq \text { const. }\left\{P_{j}(\tau)+C_{j}(\tau)+M_{j}(\tau)+\omega^{j}(\tau)\right\} .
$$

This completes the proof of the lemma.

The oscillation of a solution in a cone $K_{j}$ corresponding to a genuinely nonlinear field is essentially determined by the balance of $j$-waves in the sense given by the following lemma. We shall denote the oscillation of an arbitray function $f$ on an interval $[x, y]$ by

$$
\operatorname{osc} f[x, y]=\sup \{|f(a)-f(b)|: a, b \in[x, y]\},
$$


and the oscillation of a measure $\mu$ by

$$
\operatorname{osc} \mu[x, y]=\sup \{|\mu\{[a, b]\}|: a, b \in[x, y]\} \text {. }
$$

Lemma 4.3. Let $j$ denote the index of a genuinely nonlinear field. Then

(4.10) osc $U_{h}[x, y] \leqq$ const. $\left\{\operatorname{osc}\left(\omega_{j, h}^{+}-\omega_{j, h}^{-}\right)[x, y]+\theta_{j, h}[x, y]+\sum_{k \neq j} \omega_{k, h}[x, y]\right\}$ where the constant depends only in the $L^{\infty}$-norm of $U_{h}$.

This result is an immediate corollary of

Lemma 4.4. Let $j$ denote the index of a genuinely nonlinear field. Then

$$
\begin{aligned}
& \left|U_{h}(x, t)-U_{h}(y, t)\right| \\
& \quad \leqq \text { const. }\left\{\left|\omega_{j, h}^{+}[x, y]-\omega_{j, h}^{-}[x, y]\right|+\theta_{j, h}[x, y]+\sum_{k \neq j} \omega_{k, h}[x, y]\right\},
\end{aligned}
$$

where the constant depends only on the $L^{\infty}$-norm of $U_{h}$.

The proof of Lemma 4.4 is based on the following observation. Consider a finite sequence of constant states $U_{0}, U_{1}, \ldots, U_{n}$ such that any two consecutive states are connected by either a $j$-rarefaction wave or a $j$-compression wave. Since all of the states $U_{h}$ lie on the curve

it is clear that

$$
\frac{d u}{d \varepsilon}=r_{j}(U)
$$

$$
\left|U_{0}-U_{n}\right| \leqq \text { const. }\left|\sum_{k=1}^{n} \varepsilon_{k}\right|,
$$

where $\varepsilon_{k}$ denotes the magnitude of the $k$-th wave in the sequence. If two consecutive states are allowed to be connected by a $j$-shock wave, it is necessary to augment the right-hand side of (4.12) by the sum of the cubes of the magnitudes of all $j$-shock waves in the sequence. If, in addition, two consecutive states are allowed to be connected by an arbitrary wave, we crudely estimate their contribution by the total variation norm of all $k$-waves, $k \neq j$.

Proof of Lemma 4.4. Consider an arbitrary sequence of constant states $U_{0}, \ldots, U_{n}$. If two consecutive states $U_{l}$ and $U_{l+1}$ are connected by a $j$-wave we shall associate the magnitude of that wave with the pair $\left(U_{l}, U_{l+1}\right)$. Otherwise, we shall associate with $\left(U_{l}, U_{l+1}\right)$ the Euclidean distance $\left|U_{l}-U_{l+1}\right|$. The sequence is arbitrary in the sense that consecutive states need not be connected by an elementary wave.

The proof proceeds by induction on $n$. We shall assume that

$$
\left|U_{0}-U_{n}\right| \leqq \text { const. }\left\{\left|\sum_{j \text {-waves }} \varepsilon_{j}\right|+\sum_{j \text {-shocks }}\left|\varepsilon_{j}\right|^{3}+\Sigma\left|U_{l}-U_{l+1}\right|\right\}
$$

for all sequences which consist of at most $n$ states. The first summation on the right hand side of (4.13) consists of the magnitudes of all $j$-waves in the sequence. The second summation consists of the magnitude of $j$-shock waves only. The third summation is taken over all of the remaining pairs, i.e. pairs which are not 
connected by a $j$-wave. In the special case where the states $U_{l}$ and $U_{l+1}$ are connected by a $k$-wave, the Euclidean distance is dominated by the wave magnitude:

$$
\left|U_{l}-U_{l+1}\right| \leqq \text { const. }\left|\varepsilon_{k}\right| \text {. }
$$

The case $n=1$ is immediate. The inductive step is a consequence of the following two propositions. The constant in (4.13) for $n>1$ is selected as the maximum of the constant in (4.13) in the case $n=1$ and the constants which appear below in (4.14) and (4.15).

Proposition 1. Consider three constant states $V_{1}, V_{2}$ and $V_{3}$ such that $V_{1}$ is connected to $V_{2}$ by a j-wave of magnitude $\delta_{1}$ and $V_{2}$ is connected to $V_{3}$ by a $j$-wave of magnitude $\delta_{2}$. Then there exists a constant state $V_{4}$ such that $V_{1}$ can be connected to $V_{4}$ by a j-wave with magnitude $\delta_{1}+\delta_{2}$ and such that

$$
\left|V_{1}-V_{4}\right| \leqq \text { const. } \Sigma\left\{\left|\delta_{j}\right|^{3}: \delta_{j}<0\right\} \text {. }
$$

Proof. If neither $\delta_{1}$ nor $\delta_{2}$ is a shock wave choose $V_{4}=V_{3}$. If neither $\delta_{1}$ nor $\delta_{2}$ is a rarefaction wave choose $V_{4}$ to be that particular state on the compression wave curve through $V_{1}$ which has magnitude $\delta_{1}+\delta_{2}$. In both cases the estimate (4.14) is easily verified. Finally, suppose that $\delta_{1}$ is a shock and $\delta_{2}$ is a rarefaction wave. If $\delta_{1}+\delta_{2} \geqq 0$ then choose $V_{4}$ to be the state on the rarefaction wave curve through $V_{1}$ with magnitude $\delta_{1}+\delta_{2}$. If $\delta_{1}+\delta_{2}<0$ choose $V_{4}$ to be the state on the compression wave curve through $V_{1}$ with magnitude $\delta_{1}+\delta_{2}$. The subcase where $\delta_{1}$ is a rarefaction wave and $\delta_{2}$ a shock is handled similarly.

Proposition 2. Consider three constant states $V_{1}, V_{2}, V_{3}$ where $V_{1}$ is arbitrary and where $V_{2}$ and $V_{3}$ are connected by a j-wave of magnitude $\varepsilon$. Then there exists a constant state $V_{4}$ such that $V_{1}$ and $V_{4}$ are connected by a j-wave of magnitude $\varepsilon$ and such that

$$
\left|V_{4}-V_{3}\right| \leqq \text { const. }\left|V_{1}-V_{2}\right| \text {. }
$$

Proof. Choose $V_{4}$ to be that particular constant state which lies on the $j$-wave curve through $V_{1}$ and which has magnitude $\varepsilon$. If, for example, $V_{2}$ and $V_{3}$ are connected by a $j$-compression wave the state $V_{4}$ is chosen to lie on the $j$-compression wave curve through $V_{1}$. This completes the proof of the proposition and the lemma.

We observe that the estimates (4.10) and (4.11) also hold in the general case where the magnitude $\delta$ of an elementary $j$-wave is measured by any smooth function $z$ with the following property. The function $z$ decreases from left to right across $j$-shocks and compression waves and increases from left to right across $j$-rarefaction waves; i.e.

satisfies

$$
\delta=z\left\{U_{j}(\varepsilon)\right\}-z\left\{U_{j}(0)\right\}
$$

$$
\operatorname{sign} \delta=\operatorname{sign} \varepsilon \text {. }
$$

Fixing the coordinate function $z$, it can be further shown that any smooth function $w$ which decreases from left to right across $j$-shocks and compression waves and which increases from left to right across $j$-rarefaction waves satisfies the following 
two estimates:

$$
\begin{aligned}
w\left\{U_{h}(x, t)\right\}-w\left\{U_{h}(y, t)\right\} \leqq & \text { const. }\left\{\max \left(\omega_{j, h}^{-}[x, y]-\omega_{j, h}^{+}[x, y], 0\right)\right. \\
& \left.+\theta_{j, h}[x, y]+\sum_{k \neq j} \omega_{k, h}[x, y]\right\} \\
w\left\{U_{h}(y, t)\right\}-w\left\{U_{h}(x, t)\right\} \leqq & \text { const. }\left\{\max \left(\omega_{j, h}^{+}[x, y]-\omega_{j, h}^{-}[x, y], 0\right)\right. \\
& \left.+\theta_{j, h}[x, y]+\sum_{k \neq j} \omega_{k, h}[x, y]\right\} .
\end{aligned}
$$

Roughly speaking, the balance between $j$-waves of positive and negative magnitude as measured by any two functions with the same first order behavior is equivalent up to third order terms in $j$-shocks modulo the influence of $k$-waves, $k \neq j$.

We are now prepared to establish the decay of the oscillation of the solution. We shall consider solutions $U$ constructed as the limit of a sequence of Glimm difference approximations $U_{h}$ which satisfy local conservation laws for waves in which the error term is uniformly summable with respect to $h$ (cf. (2.11)). We further assume that the eigenvalues $\lambda_{j}$ are strictly separated on the range of the sequence of approximate solutions $U_{h}$.

Lemma 4.5. Consider a system of conservation laws in class $K_{1}$ and let $\alpha_{1}, \ldots, \alpha_{m}$ reference the linearly degenerate characteristic fields:

$$
r_{k} \cdot \nabla \lambda_{k} \equiv 0, \quad k=\alpha_{1}, \ldots, \alpha_{m} .
$$

Suppose $\phi$ is a Riemann invariant for all of the linearly degenerate fields:

$$
r_{k} \cdot \nabla \phi=0, \quad k=\alpha_{1}, \ldots, \alpha_{m} .
$$

Then $\phi\{U(x, t)\}$ satisfies the decay law

$$
\lim _{t \rightarrow \infty} \operatorname{osc} \phi\{U(\cdot, t)\}=0 .
$$

Remarks. 1. Let $\Omega$ denote the domain of definition of the nonlinear term $F$ in system (1.1). If $\Omega \neq R^{n}$ we must also assume that the range of the approximate solutions $U_{h}$ is contained in a fixed compact subset of $\Omega$. In particular, for the equations of fluid flow it is necessary to assume that the density $\rho\left(U_{h}\right)$ is bounded from below by a positive constant independent of $h$. We note that the former condition is guaranteed by the general theory provided the total variation of the initial data is chosen sufficiently small.

2. If the system (1.1) is strictly hyperbolic only on some open subset $\mathcal{O} \subset \Omega$, it is necessary to assume that the range of the approximate solutions is contained in a fixed compact subset of $\mathcal{O}$. In particular, for the equation of magnetofluid dynamics it is necessary to assume that the transverse component of the magnetic field never vanishes in the sense that

$$
H_{2, h}^{2}+H_{3, h}^{2} \geqq \text { const. }>0,
$$

where the constant is independent of $h$. We note that the former condition is guaranteed by the general theory provided the initial data have sufficiently small 
total variation. If $\mathrm{H}_{2}=\mathrm{H}_{3}=0$ the equations of magneto-fluid-dynamics formally reduce to the equations of gas dynamics.

In the proof of Lemma 4.5 we shall assume for simplicity that there exists a function $\psi$ which qualifies as a Riemann invariant for all of the linearly degenerate fields, i.e.

$$
r_{k} \cdot \nabla \psi=0, \quad k=\alpha_{1}, \ldots, \alpha_{m},
$$

and which decreases from left to right across all $j$-shock waves and increases from left to right across all $j$-rarefaction waves. The advantage of this structure is that the increasing and decreasing variations of $\psi$ are partitioned equally between $j$-shock waves and $j$-compression waves since $\psi\{U(x, t)\}$ has compact support in $x$ for each fixed $t$. We note that the equations of gas dynamics in both relativistic and nonrelativistic form as well as the equations of magneto-fluid-dynamics possess such a function $\psi$. For the former systems the fluid velocity serves as the function $\psi$. For the latter system the $x$-component of the fluid (vector) velocity serves as the function $\psi$.

For a genuinely nonlinear system of conservation laws it is easy to construct a function, $\tilde{\psi}$, which decreases from left to right across shock waves and increases from left to right across rarefaction waves, at least in the case of solutions with small oscillation. Indeed, it is sufficient to choose $\tilde{\psi}$ as an appropriate linear combination of the components of $U$ which satisfies

$$
r_{j} \cdot \nabla \tilde{\psi}>0
$$

in some neighborhood of $U$-space which contains the range of the solution.

Proof of Lemma 4.5. Fix $t$ and let $(x, t)$ and $(y, t)$ lie in the cross-section $K_{j}(t)$ of a cone $K_{j}$ corresponding to a genuinely nonlinear field. Lemma 4.2 guarantees that

$$
\lambda_{j}\{U(x, t)\}-\lambda_{j}\{U(y, t)\} \leqq \text { const. }\left\{P_{j}(t)+C_{j}(t)+M_{j}(t)+\omega^{j}(t)\right\} .
$$

Passing to the limit in (4.16) implies that

$$
\psi(x, t)-\psi(y, t) \leqq \text { const. }\left\{\max \left[\lambda_{j}(x, t)-\lambda_{j}(y, t), 0\right]+\theta_{j}[x, y]+\sum_{k \neq j} \omega_{k}[x, y]\right\}
$$

with the possible exception of countably many values of $x$ and $y$. For simplicity we have adopted the abbreviations

$$
\psi(x, t)=\psi\{U(x, t)\}, \quad \lambda_{j}(x, t)=\lambda_{j}\{U(x, t)\} .
$$

Thus if $(x, t)$ and $(y, t)$ lie in $K_{j}(t)$ we obtain from (4.18) and (4.19)

$$
\psi(x, t)-\psi(y, t) \leqq \text { const. }\left\{P_{j}(t)+C_{j}(t)+M_{j}(t)+\omega^{j}(t)+\theta_{j}[x, y]\right\} .
$$

Hence, for arbitrary $x$ and $y$

$$
\psi(x, t)-\psi(y, t) \leqq \text { const. }\left\{\Sigma P_{j}(t)+\Sigma C_{j}(t)+\Sigma M_{j}(t)+\Sigma \omega^{j}(t)\right\} .
$$

Here we have used the fact that

if $(x, t)$ and $(y, t)$ lie in $K_{j}(t)$

$$
\theta_{j}[x, y] \leqq \text { const. } M_{j}(t)
$$


Since the function $\psi$ has compact support for each fixed $t$ it follows that

$$
\operatorname{osc} \psi(\cdot, t) \leqq \text { const. }\left\{\Sigma P_{j}(t)+\Sigma C_{j}(t)+\Sigma M_{j}(t)+\Sigma \omega^{j}(t)\right\} .
$$

We conclude from Lemmas 3.1 and 4.1 that

$$
\lim _{t \rightarrow \infty} \operatorname{osc} \psi(\cdot, t)=0 \text {. }
$$

If $\phi$ is an arbitrary Riemann invariant for all of the linearly degenerate fields, inequalities (4.16) and (4.17) imply that

$$
\operatorname{osc} \phi(\cdot, t) \leqq \text { const. osc }\left\{\psi(\cdot, t)+\Sigma M_{j}(t)+\Sigma \omega^{j}(t)\right\}
$$

and therefore that $\phi$ also decays in the oscillation norm. This completes the proof of Lemma 4.5.

The case of genuinely nonlinear systems can be treated in virtually the same fashion. Consider a solution $U$ which exists as the limit of a sequence of Glimm difference approximations that satisfy local conservation laws in which the error term is uniformly summable in $h$. If the eigenvalues $\lambda_{j}$ are strictly separated on the range of the sequence of approximate solutions we obtain the following decay result.

Lemma 4.6. Consider a system of conservation laws in class $K_{2}$. Then all components of the solution decay to zero in the total variation norm:

$$
\lim _{t \rightarrow \infty} T V_{x} U(x, t)=0 \text {. }
$$

The remarks following Lemma 4.5 also apply to Lemma 4.6.

\section{Decay of the Total Variation}

For genuinely nonlinear systems of conservation laws, the decay of the total variation of the solution is essentially a consequence of two facts: the decay of the oscillation and the asymptotic uncoupling of the characteristic fields. For systems which admit linearly degenerate fields we shall establish a corresponding result that applies to functions which qualify as Riemann invariants for all the degenerate fields. In general the total variation of the solution or its distinguished components can be estimated in terms of the total variation of the eigenvalues $\lambda_{j}$. In this regard we recall that $\Omega_{j, h}(a, b, \tau)$ denotes the set of magnitudes of those $j$-waves in $U_{h}$ which intersect

$$
\{(x, t): a \leqq x<b, t=\tau\},
$$

and that the family of measures $\lambda_{j, h}=\lambda_{j, h}(\tau)$ is defined by the equation

$$
\lambda_{j, h}[a, b)=\Sigma\left\{\delta: \delta \in \Omega_{j, h}\right\} .
$$

The dependence on $\tau$ is suppressed for simplicity in printing. For a fixed value of $\tau$, the measure $\lambda_{j, h}(\tau)$ is generated by the function

$$
\tilde{\lambda}_{j, h}(x)=\tilde{\lambda}_{j, h}(x, \tau)=\lambda_{j, h}(-\infty, x)
$$


Since the total variation of the family of functions $\tilde{\lambda}_{j, h}(\cdot, \tau)$ is uniformly bounded in $h$, we may assume, after passing to a subsequence, that $\tilde{\lambda}_{j, h}(\cdot, \tau)$ converge pointwise in $x$ for any given countable set of values of $\tau$. Let

$$
\tilde{\lambda}_{j}(x)=\tilde{\lambda}_{j}(x, \tau)=\lim _{h \rightarrow 0} \tilde{\lambda}_{j, h}(x, \tau) .
$$

Our analysis of the decay of the total variation norm is based on the following lemma. Fix an interval of the form (5.1) and let $X$ and $Y$ denote a pair of generalized $j$-characteristics which pass through $(a, \tau)$ and $(b, \tau)$, respectively. Let $\pi_{\tau}$ denote the forward strip

$$
\pi_{\tau}=\{(x, t): X(t) \leqq x \leqq Y(t), t \geqq \tau\} .
$$

For a fixed value of $\tau$ let $T V \tilde{\lambda}_{j}[a, b]$ denote the total variation of the function $\tilde{\lambda}_{j}(\cdot, \tau)$ restricted to the interval $[a, b]$.

Lemma 5.1. Let $j$ denote the index of a genuinely nonlinear characteristic field. There exists a constant $T>0$ such that the interval $[T, \infty)$ contains a countable dense set of points $t$ such that for all $a$ and $b$,

$$
T V \tilde{\lambda}_{j}[a, b] \leqq \text { const. Osc }\left\{\tilde{\lambda}_{j}[a, b]+\sum_{k \neq j} \omega_{k}[a, b]+P\left(\pi_{\tau}\right)+C\left(\pi_{\tau}\right)\right\},
$$

where the constant depends only on the $L^{\infty}$-norm of the solution $U$.

Estimate (5.2) can be interpreted in the following way. Let $j$ denote the index of a genuinely nonlinear field and fix the time $t=\tau$. Suppose that the restriction of the solution $U(\cdot, \tau)$ to its elementary $j$-waves has small oscillation. Equivalently, suppose that a time $t=\tau$ the $j$-shock waves and $j$-rarefaction waves are nearly equidistributed throughout every given interval in space. In a solution with this structure the total variation of the restriction of the solution to $j$-waves is bounded by the total amount of wave interaction and cancellation plus the total strength of all $k$-waves $k \neq j$. This phenomenon is a consequence of the geometry of shock waves and rarefaction waves in the $x-t$ plane. The condition of genuine nonlinearity forces adjacent shock waves and rarefaction waves of the same field to collide and produce an amount of wave interaction and/or cancellation which increases as the total variation of the solution increases.

The main idea of the proof of Lemma 5.1 is the following. The interval $[a, b] \times$ $\{t=\tau\}$ is partitioned into a finite number of disjoint intervals which alternately contain mainly $j$-shock waves or mainly $j$-rarefaction waves; the values of $\tilde{\lambda}_{j}$ at the end points of any two adjacent intervals are approximately equal. Then we show that a given pair of adjacent subintervals will contain in general some subinterval in which the total amount of $j$-shock wave exceeds twice the total amount of $j$-rarefaction wave. The latter fact together with the condition of genuine nonlinearity guarantees that the $j$-characteristics issuing from the end points of the subinterval will coalesce in a finite time. The conservation laws for waves then imply that the total amount of $j$-rarefaction wave contained in the subinterval is bounded by the total amount of wave interaction and cancellation which occurs in the domain bounded by the $j$-characteristics issuing from its end points. We recall that generalized $j$-characteristics are constructed in such a way that they do not cross $j$-rarefaction waves. Thus, in a domain with the above 
structure the total strength of all $j$-rarefaction waves entering the domain must be balanced by the total amount of wave cancellation which occurs in the $j$-characteristic field modulo the total amount of wave interaction.

In the above construction it is essential that the total amount of $j$-shock wave strictly exceed twice the total amount of $j$-rarefaction wave. It is possible for $j$-shock waves to leave the interior of a domain bounded by a pair of $j$-characteristics by entering the one of the characteristics. This process may reduce the rate of spreading of the $j$-characteristics by approximately one-half the strength of the entering shock. The reduction follows from the fact that the speed of propagation of a generalized $j$-characteristic is approximately equal to the average value of the characteristic speeds $\lambda_{j}$ on both sides of the characteristic [17].

Before providing the details of the proof of Lemma 5.1 we shall state and prove our main results on decay in the total variation norm. Consider a solution $U$ constructed as the limit of a sequence of Glimm difference approximations $U_{h}$ that satisfy local conservation laws for waves in which the error term is uniformly summable with respect to $h$ cf. $((2.11))$. Suppose further that the eigenvalues $\lambda_{j}$ are strictly separated on the range of the sequence of approximate solutions $U_{h}$.

Theorem 5.1. Consider a system of conservation laws in class $K_{1}$ and suppose $\phi$ is a Riemann invariant for all of the linearly degenerate characteristic fields. Then $\phi\{U(x, t)\}$ satisfies the decay law

$$
\lim _{t \rightarrow \infty} T V_{x} \phi\{U(x, t)\}=0 .
$$

Theorem 5.2. Consider a system of conservation laws in class $K_{2}$. The solution $U(x, t)$ satisfies the decay law

$$
\lim _{t \rightarrow \infty} T V_{x} U(x, t)=0 .
$$

Remark. The remarks following Lemma 4.5 also apply to Theorems 5.1 and 5.2 .

Proof of Theorem 5.1. We need only estimate the total variation of $\phi$ at a dense set of times since the solution is $L^{1}$-continuous in time [9]:

$$
\int_{-\infty}^{\infty}\left|U\left(x, t_{1}\right)-U\left(x, t_{2}\right)\right| d x \leqq \text { const. }\left|t_{1}-t_{2}\right| .
$$

Fix an index $j$ corresponding to a genuinely nonlinear field. Estimates (4.16) and (4.17) imply that

$$
\left|\phi\left\{U_{h}(x, t)\right\}-\phi\left\{U_{h}(y, t)\right\}\right| \leqq \text { const. }\left\{\left|\tilde{\lambda}_{j, h}(x, t)-\tilde{\lambda}_{j, h}(y, t)\right|+\theta_{j, h}[x, y]+\sum_{k \neq j} \omega_{k, h}[x, y]\right\} .
$$

Passing to the limit we obtain

$$
|\phi\{U(x, t)\}-\phi\{U(y, t)\}| \leqq \text { const. }\left\{\left|\tilde{\lambda}_{j}(x, t)-\tilde{\lambda}_{j}(y, t)\right|+\theta_{j}[x, y]+\sum_{k \neq j} \omega_{k}[x, y]\right\}
$$

for almost all $x$ and $y$. Thus,

$$
T V \phi[a, b] \leqq \text { const. }\left\{T V \tilde{\lambda}_{j}[a, b]+\theta_{j}[a, b]+\sum_{k \neq j} \omega_{k}[a, b]\right\}
$$


at rational values of $t$. Estimate (5.2) then yields

$$
T V \phi[a, b] \leqq \text { const. }\left\{\text { Osc } \tilde{\lambda}_{j}[a, b]+P(\pi)+C(\pi)+\theta_{j}[a, b]+\sum_{k \neq j} \omega_{k}[a, b]\right\} .
$$

The first four terms on the right-hand side of inequality (5.5) approach zero as $t$ approaches infinity for arbitrary values of $a$ and $b$. If $a$ and $b$ are restricted to lie in the cross-section $K_{j}(t)$ of the cone $K_{j}$ then the fifth term also approaches zero by Lemma 4.1 . We conclude that the total variation of $\phi$ restricted to the crosssection $K_{j}(t)$ decays to zero with large time.

Since $\phi$ is continuous across $k$-contact discontinuities the total variation of its restriction to cones $K_{k}$ corresponding to linearly degenerate fields is of the order

$$
\sum_{l \notin \alpha} \omega_{l}\left\{K_{k}(t)\right\}
$$

and thus decays to zero with large time by Lemma 4.1 . Here $\alpha=\left\{\alpha_{1}, \ldots, \alpha_{m}\right\}$ denotes the set of indices of the linearly degenerate fields. This completes the proof of Theorem 5.1 .

The proof of Theorem 5.2 is similar and follows from the estimate

$$
|U(x, t)-U(y, t)| \leqq \text { const. }\left\{\left|\tilde{\lambda}_{j}(x, t)-\tilde{\lambda}_{j}(y, t)\right|+\theta_{j}[x, y]+\sum_{k \neq j} \omega_{k}[x, y]\right\}
$$

We omit the details.

We shall partition the proof of Lemma 5.1 into three propositions. Let $f=f(x)$ be a function of bounded variation which is defined on the interval $[a, b)$. Let $f^{ \pm}$ denote the positive and negative variation measures associated with $f$ :

$$
f(x)=f^{+}\{[a, x)\}-f^{-}\{[a, x)\} .
$$

Proposition 5.1. Suppose that the positive variation measure $f^{+}$does not contain any point masses. Fix a constant $M>1$. Then for every $\varepsilon>0$ there exists a finite collection of disjoint subintervals $\Omega_{j}$ of $[a, b)$ such that

$$
\begin{gathered}
\sum_{j}\left|f^{-}\left\{\Omega_{j}\right\}-M f^{+}\left\{\Omega_{j}\right\}\right| \leqq \varepsilon \\
f^{+}\left\{[a, b)-\bigcup \Omega_{j}\right\} \leqq \text { const. }\left[f^{+}\left\{\bigcup \Omega_{j}\right\}+\operatorname{osc} f+\varepsilon\right],
\end{gathered}
$$

where the constant depends only on $M$.

The application of Proposition 5.1 to the function $\tilde{\lambda}_{j}(\cdot, \tau)$ yields the existence of a collection of subintervals on which the total strength of all $j$-waves of negative magnitude exceeds three times the total strength of all $j$-waves of positive magnitude. The choice $M=3$ can be replaced by any constant greater than two. The union of the intervals $\Omega_{j}$ accounts for a uniform fraction of the total variation of $f$, modulo a quantity of the order of the oscillation of $f$ (cf. (5.7)).

Proof of Proposition 5.1. Since the measures $f^{+}$and $f^{-}$are mutually singular, there exists a finite collection of subintervals $I_{k}^{\prime}=\left[c_{k}, d_{k}\right.$ ) having disjoint closures such that

$$
f^{+}\left\{\bigcup I_{k}^{\prime}\right\}+f^{-}\left\{I-\bigcup I_{k}^{\prime}\right\} \leqq \varepsilon
$$


We may assume without loss of generality that $I=\left[c_{0}, c_{n}\right)$ : for example, if $a<c_{0}$ then (5.8) implies that

$$
f^{-}\left\{\left[a, c_{0}\right)\right\} \leqq \varepsilon .
$$

Thus, we may assume that $I$ is partitioned into $2 n$ disjoint intervals:

where the intervals $I_{k}$ have the form

$$
I=\bigcup I_{k}
$$

$$
\begin{aligned}
I_{2 k} & =\left[d_{k-1}, c_{k}\right), & & k=1,2, \ldots, n \\
I_{2 k+1} & =\left[c_{k}, d_{k}\right), & k & =0,1, \ldots, n-1 .
\end{aligned}
$$

We observe that the measure $f^{-}$is essentially concentrated on the intervals with even index and $f^{-}$on the intervals with odd index:

where $f_{j}^{ \pm}=f^{ \pm}\left(I_{j}\right)$.

$$
\Sigma f_{2 k+1}^{+}+\Sigma f_{2 k}^{-} \leqq \varepsilon
$$

Next we define by induction a certain collection of intervals $J_{k}$. The required intervals $\Omega_{k}$ will be defined as certain subintervals of $J_{k}$. Let

$$
J_{1}=\bigcup_{1}^{2 p} I_{k}
$$

where $2 p$ is the smallest even index such that

$$
\sum_{1}^{2 p-1} f_{2 k+1}^{-} \leqq M \sum_{2}^{2 p} f_{2 k}^{+}
$$

here the summation on the left is taken over all odd indices between 1 and $2 p-1$ and the summation on the right is taken over all even indices between 2 and $2 p$. If no such index $2 p$ exists we define $J_{1}=I$. Assuming that the intervals $J_{k}$ have been defined for $k \leqq m-1$ and that each is the union of an even number of intervals $I_{k}$, we define $J_{m}$ as follows. Let $2 l$ be the unique index such that

We define

$$
\bigcup_{1}^{m-1} J_{k}=\bigcup_{1}^{2 l} I_{k}
$$

$$
J_{m}=\bigcup_{2 l+1}^{2 q} I_{k}
$$

where $2 q$ is the smallest even index greater than $2 l$ such that

$$
\sum_{2 l+1}^{2 q-1} f_{2 k+1}^{-} \leqq M \sum_{2 l}^{2 q} f_{2 k}^{+} .
$$

If no such index $2 q$ exist we define

$$
J_{m}=\bigcup_{2 l+1}^{2 n} I_{k}
$$

Without loss of generality we may assume the following two conditions. First, the interval $J_{1}$ does not equal $I$. Otherwise

$$
\Sigma f_{k}^{+}+f_{k}^{-} \leqq \text {const. }\{\operatorname{osc} f+\varepsilon\},
$$


where the summations are taken over all indices $k$ and where the constant depends only on $M$. Estimate (5.13) can be established as follows. If $J_{1}=I$ then

and we see from (5.8) that

$$
\sum_{2}^{2 n} f_{2 k}^{+}<\frac{1}{M} \sum_{1}^{2 n-1} f_{2 k+1}^{-},
$$

$$
\Sigma f_{k}^{+} \leqq \frac{1}{M} \Sigma f_{k}^{-}+2 \varepsilon
$$

Since

$$
\left|f_{k}^{+}-\Sigma f_{k}^{-}\right| \leqq \operatorname{osc} f
$$

it follows from (5.14) that

and therefore that

$$
\Sigma f_{k}^{+} \leqq \frac{1}{M} \Sigma f_{k}^{+}+\frac{1}{M} \text { osc } f+2 \varepsilon
$$

$$
\Sigma f_{k}^{+} \leqq \frac{1}{M-1} \text { osc } f+\frac{2 \varepsilon M}{M-1} .
$$

The estimate (5.13) follows from (5.15). For similar reasons we may also assume that our inductive construction yields a collection of intervals $J_{k}, k=1,2, \ldots r$, where $J_{r}$ has the form

and

$$
J_{r}=\sum_{2}^{2 n} I_{k}
$$

Fix an interval

$$
\sum_{2 s+1}^{2 n-1} f_{2 k+1}^{-} \leqq M \sum_{2 s}^{2 n} f_{2 k}^{+}
$$

$$
J_{j}=\sum_{2+1}^{2 t} I_{k}
$$

Since $f^{+}$does not contain any point masses it follows from the definition of $J_{j}$ that there exists a point $y_{j}$ such that

where

$$
\sum_{2 s+1}^{2 t-1} f^{-}\left(I_{2 k+1}\right)=M\left\{\sum_{2 s+2}^{2 t-2} f^{+}\left(I_{2 k}\right)+f^{+}\left(\tilde{I}_{2 t}\right)\right\}
$$

$$
\tilde{I}_{2 t}=I_{2 t}\left\{x: x \leqq y_{j}\right\}
$$

We note that the right hand side will contain only the term $f^{+}\left(\tilde{I}_{2 t}\right)$ in the case where $2 t-2<2 s+2$. We define

$$
\Omega_{j}=J_{j} \cap\left\{x: x \leqq y_{j}\right\} .
$$

The estimate (5.7) follows from the facts that

and

$$
\left|\Sigma f^{+}\left\{\Omega_{j}\right\}+\Sigma f^{+}\left\{J_{j}-\Omega_{j}\right\}-\Sigma f^{-}\left\{J_{j}\right\}\right| \leqq \operatorname{osc} f+\varepsilon
$$

$$
f^{-}\left\{J_{j}\right\}=M f^{+}\left\{\Omega_{j}\right\}+\varepsilon_{j}
$$

where $\Sigma \varepsilon_{j} \leqq \varepsilon$. This completes the proof of the proposition. 
Proposition 5.2. Let $j$ denote the index of a genuinely nonlinear characteristic field. There exists an interval $[T, \infty)$ almost all of whose points $\tau$ have the following property. If, for a given subsequence $h_{k}$, the limit

$$
\phi_{j}(x, \tau) \stackrel{\text { def }}{=} \lim _{h_{k} \rightarrow 0} \tilde{\lambda}_{j, h_{k}}(x, \tau)
$$

exists for almost all $x$, then the positive variation measure associated with the function $\phi_{j}(\cdot, \tau)$ does not contain any point masses.

Sketch of proof. Let $\Gamma$ denote the set of jump points of $U$ considered as a function of bounded variation in the sense of Cesari [6], [26]. Evaluating the measure

$$
\frac{\partial}{\partial t} U+\frac{\partial}{\partial x} F(U)
$$

on an arbitrary measurable subset of $\Gamma$ implies that the Rankine-Hugoniot relations are satisfied at almost all points $P$ of $\Gamma$ with respect to one-dimensional Hausdorff measure:

$$
v_{t}\left\{l_{v} U-l_{-v} U\right\}+v_{x}\left\{F\left(l_{v} U\right)-F\left(l_{-v} U\right)\right\}=0 .
$$

Here $v=\left(v_{x}, v_{t}\right)$ denotes the normal to $\Gamma$ and $l_{ \pm v} U=l_{ \pm v} U(P)$ denote the approximate limits of $U$ with respect to the complementary half-planes through $P$ defined by $v[26]$. Evaluating the measure

$$
\frac{\partial}{\partial t} \eta(U)+\frac{\partial}{\partial x} q(U)
$$

on an arbitrary measurable subset of $\Gamma$ implies that

$$
v_{t}\left\{\eta\left(l_{v} U\right)-\eta\left(l_{-v} U\right)\right\}+v_{x}\left\{q\left(l_{v} U\right)-q\left(l_{-v} U\right)\right\} \leqq 0
$$

at almost all points of $\Gamma$. Thus, if $l_{ \pm \nu} U$ are sufficiently close, the Lax shock conditions

$$
\lambda_{k}\left\{l_{v} U\right\}<-v_{t} / v_{x}<\lambda_{k}\left\{l_{-\nu} U\right\}
$$

are satisfied for a unique index $k$. Here we have normalized the direction of $v$ by assuming $v_{x}<0$.

Now, for almost all values of $\tau$ in the sense of Lebesque measure, the points of discontinuity of the restriction of $U$ to $t=\tau$ are jump points at which the onesided limits of the restriction $U(\cdot, \tau)$ equal the corresponding approximate limits:

$$
U(x \pm 0, \tau)=l_{ \pm v} U(x, \tau)
$$

Thus, each point of discontinuity of the restriction $U(\cdot, \tau)$ satisfies

$$
\lambda_{k}\{U(x+0, \tau)\}<-v_{t} / v_{x}<\lambda_{k}\{U(x-0, \tau)\}
$$

for a unique index $k$.

Suppose, on the contrary, that $\phi^{+}(\cdot, \tau)$ has a point mass at the point $x_{0}$. Then there exists a constant $\alpha>0$ with the following property. For all sufficiently small $\delta>0$ there exists a constant $H(\delta)$ such that

$$
\left.\tilde{\lambda}_{j, h_{k}}^{+}\left\{\left[x_{0}-\delta, x_{0}+\delta\right]\right\} \geqq \tilde{\lambda}_{j, h_{k}}\left[x_{0}-\delta, x_{0}+\delta\right]\right\}+\alpha
$$


provided $h_{k} \leqq H(\delta)$. Since the interaction measure $Q$ has at most countably many point masses, we may assume without loss of generality that the line $t=\tau$ does not contain any point masses of $Q$. Thus, in the limit as $h_{k}$ approaches zero, the total strength of all $k$-waves, $k \neq j$, in $U_{h}$ which cross $\left[x_{0}-\delta, x_{0}+\delta\right]$ at time $t=\tau$ is arbitrarily small. From this fact we conclude that

$$
\phi_{j}\left(x_{0} \pm 0, \tau\right)=\lambda_{j}\left\{U\left(x_{0} \pm 0, \tau\right)\right\} .
$$

Since $\phi_{j}^{+}$is assumed to have a point mass at $x_{0}$ it follows from (5.17) that

$$
\lambda_{j}\left\{U\left(x_{0}+0, \tau\right)\right\}>\lambda_{j}\left\{U\left(x_{0}-0, \tau\right)\right\} .
$$

But (5.18) is a contradiction to the Lax shock conditions. Indeed, if $\tau$ is choosen sufficiently large the oscillation of $\phi_{j}$ can be made arbitrarily small and it follows that $U\left(x_{0}+0, \tau\right)$ lies on the $j$-shock wave through $U\left(x_{0}-0, \tau\right)$, i.e. inequality (5.16) holds with $k \neq j$. This completes the proof of the proposition.

Propositions 5.1 and 5.2 together with Proposition 5.3 below constitute the proof of Lemma 5.1. Before stating the third proposition we recall certain facts concerning generalized characteristics. Let $j$ denote the index of a genuinely nonlinear field. Consider the family of approximate solutions $U_{h}$. By passing to a subsequence we may assume that a generalized $j$-characteristic $X_{j}=X_{j}(t ; P)$ passes through each point $P$ with rational coordinates and furthermore that $X_{j}$ exists as the limit of approximate $j$-characteristics $X_{j, h}$ in $U_{h}$. We recall from [10] that the approximate solutions $U_{h}$ are uniformly equicontinuous along approximate $j$ characteristics in the following sense. Each sequence of approximate characteristics contains a subsequence, say $X_{j, k}$, with the following property. For every $\varepsilon>0$ there exists a constant $\delta(\varepsilon)$ such that

$$
\begin{aligned}
& \left|U_{k}\left\{X_{j, k}(\tau)+0, \tau\right\}-U_{k}\left\{X_{j, k}(\tau)+\delta, \tau\right\}\right| \leqq \varepsilon \\
& \left|U_{k}\left\{X_{j, k}(\tau)-0, \tau\right\}-U_{k}\left\{X_{j, k}(\tau)-\delta, \tau\right\}\right| \leqq \varepsilon
\end{aligned}
$$

with the possible exception of an at most countable set of values of $\tau$ provided $\delta \leqq \delta(\varepsilon)$. Thus, for the aforementioned family of $j$-characteristics through rational points $P$, we may assume that estimates (5.19) and (5.20) are violated at most at a countable set of values of $\tau$. This fact together with Proposition 5.2 guarantees the existence of an interval $\left[\tau^{*}, \infty\right)$ which contains a countable dense subset $C$ of points $\tau$ with the following two properties. First, the sequence of functions $\tilde{\lambda}_{j, k}(x, \tau)$ converges pointwise in $x$ to functions

$$
\tilde{\lambda}_{j}(x, \tau)=\lim _{h \rightarrow 0} \tilde{\lambda}_{j, h}(x, \tau)
$$

whose positive variation measures do not admit any point masses. Second, at time $t=\tau$ the approximate solutions $U_{h}$ are uniformly equicontinuous along the corresponding famlies of approximate characteristics $X_{j, h}(t ; P)$.

Proposition 5.3. Let $j$ denote the index of a genuinely nonlinear characteristic field. If $\tau$ is sufficiently large the functions $\tilde{\lambda}_{j}(\cdot, \tau)$ defined by (5.21) have the property that if

$$
\tilde{\lambda}_{j}^{-}[a, b] \geqq 3 \tilde{\lambda}_{j}^{+}[a, b]
$$


then

$$
\tilde{\lambda}_{j}^{+}[a, b] \leqq \text { const. }\left\{P\left(\pi_{\tau}\right)+C\left(\pi_{\tau}\right)\right\},
$$

where $\pi_{\tau}$ denotes the forward strip bounded by a pair of $j$-characteristics $X$ and $Y$ passing through $(a, \tau)$ and $(b, \tau)$, respectively:

$$
\pi_{\tau}=\{(x, t): X(t) \leqq x \leqq Y(t), t \geqq \tau\} .
$$

Proof. We may assume without loss of generality that $a$ and $b$ lie in a countable dense subset of the real numbers. The rate of spreading of the characteristics $X$ and $Y$ is given by

$$
\begin{aligned}
Y(t)-X(t)= & \lambda_{j}\{U(Y(t)+0, t)\}-\lambda_{j}\{U(X(t)-0, t)\} \\
& +\left\{\frac{1}{2}+O(\varepsilon)\right\}\{\operatorname{str} X(t)+\operatorname{str} Y(t)\},
\end{aligned}
$$

where $\varepsilon$ denotes the maximum strength of characteristics $X$ and $Y$. Let $\mu_{k, h}(t)$ denote the total amount of $k$-wave, $k \neq j$, which is contained in $U_{h}$ between the approximate characteristics $X_{h}$ and $Y_{h}$ at time $t$ :

$$
\mu_{k, h}(t)=\omega_{k, h}\left\{\left[X_{h}(t), Y_{h}(t)\right]\right\}, \quad k \neq j .
$$

Let $\mu_{j, h}^{ \pm}(t)$ denote the corresponding quantities for $j$-waves as measured by the positive and negative variation measures of $\lambda_{j, h}$ :

$$
\mu_{j, h}^{ \pm}(t)=\lambda_{j, h}^{ \pm}\left\{\left[X_{h}(t), Y_{h}(t)\right]\right\} .
$$

Using (5.23) and (5.24) we may rewrite equation (5.22) in the form

where

$$
Y(t)-X(t)=\mu_{j}^{+}(t)-\mu_{j}^{-}(t)+\left\{\frac{1}{2}+O(\varepsilon)\right\}\{\operatorname{str} X(t)+\operatorname{str} Y(t)\}+O\left\{\sum_{k \neq j} \mu_{k}(t)\right\},
$$

$$
\mu_{k}(t)=\lim _{h \rightarrow 0} \mu_{k, h}(t), \quad \mu_{j}^{ \pm}(t)=\lim _{h \rightarrow 0} \lambda_{j, h}^{ \pm}(t) .
$$

The limits (5.25) are known to exist with the possible exception of at most countably many values of $t[10]$.

Furthermore, it has been shown by GLIMM \& LAX [10] that the distance between characteristics,

$$
D(t)=Y(t)-X(t)
$$

satisfies an integral inequality of the form

$$
D(t) \leqq F(t)+\int_{t_{1}}^{t} D(s) d m(s),
$$

where

$$
\begin{aligned}
& F(t)=\text { const. } D\left(t_{1}\right)+\int_{t_{1}}^{t} \Omega(t) d t \\
& \Omega(t)=\mu_{j}^{+}(t)-\mu_{j}^{-}(t)+\left\{\frac{1}{2}+O(\varepsilon)\right\}\{\operatorname{str} X(t)+\operatorname{str} Y(T)\}
\end{aligned}
$$

and where the monotone function $m(s)$ denotes the total strength of all $k$-waves, $k \neq j$, which cross the characteristics $X$ and $Y$ in the time interval $[t, s]$ plus the quantity

$$
P\left[\left\{(x, t): X(t) \leqq x \leqq Y(t): t_{1} \leqq t \leqq s\right\}\right] .
$$


The proof of (5.26) is given in [10] for genuinely nonlinear systems of two conservation laws but applies with only very minor modifications to the genuinely nonlinear fields of an arbitrary systems of $n$ conservation laws. We omit the details.

Using the fact that $D(t)$ is bounded from above by the solution of the corresponding integral equation, we obtain the estimate

Therefore,

$$
D(t) \leqq e^{m(t)} F\left(t_{1}\right)+\int_{t_{1}}^{t} e^{m(t)-m\left(t_{1}\right)} \frac{d F}{d t} d t .
$$

$$
D(t) \leqq \text { const. } D\left(t_{1}\right)+\int_{t_{1}}^{t} e^{m(t)-m\left(t_{1}\right)} \Omega(t) d t .
$$

By estimating the integrand in the right hand side of (5.27) we shall show that the characteristic $X$ and $Y$ will coalesce in general after a finite time. In this regard we may restrict our attention to values of $t$ in a countable dense subset since the function $\Omega(t)$ is a function of bounded variation. Now, if $t \in C$ we have

$$
\Omega(t)=\tilde{\lambda}_{j}\{Y(t)+0, t\}-\tilde{\lambda}_{j}\{X(t)-0, t\}+\left\{\frac{1}{2}+O(\varepsilon)\right\}\{\operatorname{str} X(t)+\operatorname{str} Y(t)\}
$$

and

$$
\begin{aligned}
& \operatorname{str} X(t)=\tilde{\lambda}_{j}\{X(t)-0, t\}-\tilde{\lambda}_{j}\{X(t)+0, t\} \\
& \operatorname{str} Y(t)=\tilde{\lambda}_{j}\{Y(t)-0, t\}-\tilde{\lambda}_{j}\{Y(t)+0, t\},
\end{aligned}
$$

as a consequence of the equicontinuity of the approximate solutions along the approximate characteristics $X_{h}$ and $Y_{h}$. Equation (5.28) can also be written in the form

$$
\Omega(t)=\tilde{\lambda}_{j}^{+}[X(t), Y(t)]-\tilde{\lambda}_{j}^{-}[X(t), Y(t)]+\left\{\frac{1}{2}+O(\varepsilon)\right\}\{\operatorname{str} X(t)+\operatorname{str} Y(t)\} .
$$

From equation (5.29) we obtain the crude estimate

$$
\Omega(t) \leqq \tilde{\lambda}_{j}[X(t), Y(t)]-\left\{\frac{1}{2}+O(\varepsilon)\right\} \tilde{\lambda}_{j}^{-}[X(t), Y(t)] .
$$

The quantities on the right hand side of (5.30) can be related back to the corresponding values at time $t=\tau$ with the aid of the conservation laws for waves. By considering the approximate conservation laws for waves as applied to strips bounded by approximate $j$-characteristics and by passing to the limit one can establish the following estimates in a straightforward way:

$$
\begin{aligned}
& \tilde{\lambda}_{j}^{+}[X(t), Y(t)]=\tilde{\lambda}_{j}^{+}[a, b]+O\left\{C\left(\pi_{\tau}^{t}\right)+P\left(\pi_{\tau}^{t}\right)\right\} \\
& \tilde{\lambda}_{j}^{-}[X(t), Y(t)] \geqq \tilde{\lambda}_{j}^{-}[a, b]+O\left\{C\left(\pi_{\tau}^{t}\right)+P\left(\pi_{\tau}^{t}\right)\right\},
\end{aligned}
$$

where

$$
\pi_{\tau}^{t}=\{(x, s): X(s) \leqq x \leqq Y(s) ; \tau \leqq s \leqq t\} .
$$

The estimate (5.31) is simply the statement that the total strength of $j$-rarefaction waves (as measured by $\tilde{\lambda}_{j}^{+}$) is conserved up to terms of the order of the total amount of wave interaction and cancellation. The inequality in (5.32) is due to the fact that $j$-shock waves may enter the strip $\pi_{\tau}^{t}$ from the outside and become part of its lateral boundary. 
Combining estimates (5.30)-(5.32), we obtain

$$
\Omega(t) \leqq \tilde{\lambda}_{j}[a, b]-\left\{\frac{1}{2}+O(\varepsilon)\right\} \tilde{\lambda}_{j}^{-}[a, b]+K\left\{C\left(\pi_{\tau}^{t}\right)+P\left(\pi_{\tau}^{t}\right)\right\},
$$

where $K$ is an appropriate constant. Now let us suppose that

$$
\tilde{\lambda}_{j}^{-}[a, b] \geqq 3 \tilde{\lambda}_{j}^{+}[a, b] .
$$

This hypothesis implies that

$$
\Omega(t) \leqq\left\{-\frac{1}{2}+O(\varepsilon)\right\} \tilde{\lambda}_{j}^{+}[a, b]+K\left\{C\left(\pi_{\tau}\right)+P\left(\pi_{\tau}\right)\right\} .
$$

In estimate (5.33) we have used the fact that $\pi_{\tau}^{t} \subset \pi_{\tau}$. Clearly we may assume that $\tilde{\lambda}_{j}^{+}[a, b]>0$. In this situation we see that either

or

$$
\tilde{\lambda}_{j}^{+}[a, b] \leqq 2 K^{\prime}\left\{C\left(\pi_{\tau}\right)+P\left(\pi_{\tau}\right)\right\}
$$

where

$$
\Omega(t) \leqq-\tilde{\lambda}_{j}^{+}[a, b]<0,
$$

$$
K^{\prime} \stackrel{\text { def }}{=} K /\left(\frac{1}{2}+O(\varepsilon)\right) \text {. }
$$

In the former case the proof of the proposition is complete. In the latter case the $j$-characteristics $X$ and $Y$ necessarily intersect after a finite time. We may thus conclude in this case from the conservation laws for $j$-rarefaction waves that

$$
\lambda_{j}^{+}[a, b] \leqq \text { const. }\left\{C\left(\pi_{\tau}\right)+P\left(\pi_{\tau}\right)\right\} .
$$

This completes the proof of the proposition.

Note. This research was supported in part by NSF grant MPS 75-06999.

\section{References}

1. BaKhraRov, N., On the existence of regular solutions in the large for quasilinear hyperbolic systems, Zhur. Vychisl. Mat. i Mathemat. Fiz., 10, 969-980 (1970).

2. Courant, R. \& K.O.Friedrichs, "Supersonic Flow and Shock Waves", New York: Interscience Publishers, Inc., 1948.

3. DAFERMOS, C.M., private communications.

4. DAFERmos, C. M., The entropy rate admissibility criterion for solutions of hyperbolic conservation laws, J. Differential Eq., 14, 202-212 (1973).

5. DiPerna, R.J., Global solutions to a class of nonlinear hyperbolic systems of equations, Comm. Pure Appl. Math., 26, 1-28 (1973).

6. Federer, H., "Geometric Measure Theory." New York: Springer 1969.

7. Friedrichs, K.O., On the laws of relativisitc electro-magneto-fluid dynamics, Comm. Pure Appl. Math., 27, 749-808 (1974).

8. Friedrichs, K.O. \& P.D. LAx, Systems of conservation laws with a convex extension, Proc. Nat. Acad. Sci. USA 68, 1686-1688 (1971).

9. Glimm, J., Solutions in the large for nonlinear hyperbolic systems of equations, Comm. Pure Appl. Math. 18,697-715 (1965).

10. Glimm, J. \& P.D. LaX, Decay of solutions of systems of nonlinear hyperbolic conservation laws, Amer. Math. Soc., 101 (1970).

11. Gogosov, V.V., Resolution of an arbitrary discontinuity in magnetohydrodynamics, J. Appl. Math. Mech., 25, 148-197 (1961).

12. Greenberg, J.M., Estimates for fully developed shock solutions to the equation $\partial u / \partial t-\partial v / \partial x=0$ and $\partial v / \partial t-\partial \sigma(u) / x$, Indiana Univ. Math. J., 22, 989-1003 (1973). 
13. GreEnBerg, J. M., Decay theorems for stopping shock problems, preprint.

14. Jeffrey, A. \& T. TaniUti, "Non-linear Wave Propagation with Applications to Physics and Magnetohydrodynamics. New York: Academic Press 1964.

15. JoHN, F., Formation of singularities in one-dimensional nonlinear wave propagation, Comm. Pure Appl. Math., 27, 377-405 (1974)

16. Kuznetsov, N. N. \& V.A. TupshiEv, A certain generalization of a theorem of Glimm, Dokl. Akad. Nauk. SSSR, 221, 287-290 (1975).

17. LAX, P.D., Hyperbolic systems of conservation laws II, Comm. Pure Appl. Math., 10, 537-566 (1957).

18. LAX, P.D., Shock waves and entropy, "Contributions to Nonlinear Functional Analysis", ed. E. A. Zarantonello, 603-634. New York: Academic Press 1971.

19. LAx, P. D., The formation and decay of shock waves, Amer. Math. Monthly, 79, 227-241 (1972).

20. LIU, T. P., Solutions in the large for the nonisentropic equations of gas dynamics, preprint.

21. Nishida, T., Global solutions for an initial boundary value problem of a quasilinear hyperbolic system, Proc. Japan Acad., 44, 642-646 (1968)

22. Nishida, T. \& J.A.Smoller, Solutions in the large for some nonlinear hyperbolic conservation laws, Comm. Pure Appl. Math., 26, 183-200 (1973).

23. Smoller, J.A., On the solution of the Riemann problem with general step data for an extended class of hyperbolic systems, Mich. Math. J., 16, 201-210 (1969).

24. Smoller, J.A. \& J.L. Jonson, Global solutions for an extended class of hyperbolic systems of conservation laws, Arch. Rational Mech. Anal.. 37. pp. 399-400 (1970).

25. TAUb, A.H., Relativistic Rankine-Hugoniot equations, Phys. Rev., 74, 328-334 (1948).

26. VOL'PERT, A.I., the spaces BV and quasilinear equations, Math. USSR Sb., 2, 257-267 (1967).

Department of Mathematics University of Michigan Ann Arbor, Michigan

(Received June 20, 1976) 